\title{
Anticancer kinase inhibitors impair intracellular viral trafficking and exert broad-spectrum antiviral effects
}

\author{
Elena Bekerman, ${ }^{1}$ Gregory Neveu, ${ }^{1}$ Ana Shulla, ${ }^{2}$ Jennifer Brannan, ${ }^{3}$ Szu-Yuan Pu, ${ }^{1}$ Stanley Wang, ${ }^{1}$ Fei Xiao, ${ }^{1}$ Rina Barouch-Bentov, \\ Russell R. Bakken, ${ }^{3}$ Roberto Mateo, ${ }^{4}$ Jennifer Govero, ${ }^{5}$ Claude M. Nagamine, ${ }^{6}$ Michael S. Diamond, ${ }^{5}$ Steven De Jonghe, ${ }^{7}$ \\ Piet Herdewijn, ${ }^{7}$ John M. Dye, ${ }^{3}$ Glenn Randall, ${ }^{2}$ and Shirit Einav ${ }^{1}$

\begin{abstract}
Department of Medicine, Division of Infectious Diseases and Geographic Medicine, and Department of Microbiology and Immunology, Stanford University School of Medicine, Stanford, California, USA. 2Department of Microbiology, University of Chicago, Chicago, Illinois, USA. ${ }^{3}$ US Army Medical Research Institute of Infectious Diseases, Viral Immunology Branch, Frederick, Fort Detrick, Maryland, USA. ${ }^{4}$ Department of Genetics and Department of Microbiology and Immunology, Stanford University School of Medicine, Stanford, California, USA. ${ }^{5}$ Departments of Medicine, Molecular Microbiology, and Pathology and Immunology, Washington University School of Medicine, St. Louis, Missouri, USA. ${ }^{6}$ Department of Comparative Medicine, Stanford University School of Medicine, Stanford, California, USA. 'Laboratory of Medicinal Chemistry, Rega Institute for Medical Research, KU Leuven, Leuven, Belgium.
\end{abstract}

\begin{abstract}
Global health is threatened by emerging viral infections, which largely lack effective vaccines or therapies. Targeting host pathways that are exploited by multiple viruses could offer broad-spectrum solutions. We previously reported that AAK1 and CAK, kinase regulators of the host adaptor proteins AP1 and AP2, are essential for hepatitis C virus (HCV) infection, but the underlying mechanism and relevance to other viruses or in vivo infections remained unknown. Here, we have discovered that AP1 and AP2 cotraffic with HCV particles in live cells. Moreover, we found that multiple viruses, including dengue and Ebola, exploit AAK1 and GAK during entry and infectious virus production. In cultured cells, treatment with sunitinib and erlotinib, approved anticancer drugs that inhibit AAK1 or GAK activity, or with more selective compounds inhibited intracellular trafficking of HCV and multiple unrelated RNA viruses with a high barrier to resistance. In murine models of dengue and Ebola infection, sunitinib/erlotinib combination protected against morbidity and mortality. We validated sunitinib- and erlotinib-mediated inhibition of AAK1 and GAK activity as an important mechanism of antiviral action. Additionally, we revealed potential roles for additional kinase targets. These findings advance our understanding of virus-host interactions and establish a proof of principle for a repurposed, host-targeted approach to combat emerging viruses.
\end{abstract}

\section{Introduction}

A major threat to human health is posed by emerging viruses, such as dengue (DENV) and Ebola (EBOV). Dengue is estimated to infect 390 million people annually in over 100 countries (1). Dengue fever can progress to a life-threatening disease, known as severe dengue, particularly upon a secondary infection with a heterologous DENV strain. Consequently, development of a dengue vaccine has been hampered by the necessity to generate simultaneous protection against 4 distinct DENV serotypes (2). As a further challenge, recent studies have suggested that preexisting DENV immunity may enhance Zika virus (ZIKV) infection and vice versa, and consequently increase disease severity (3-5). While an Ebola vaccine has shown promise recently (6), it is not yet approved. Moreover, no effective antiviral treatment is available against DENV, EBOV, ZIKV, and most other emerging viral pathogens, leaving the global population at risk for significant morbidity and mortality.

Most antiviral therapies approved to date target viral enzymes (e.g., protease or polymerase) via a "one drug, one bug" approach.

Authorship note: E. Bekerman and G. Neveu contributed equally to this work. Conflict of interest: The authors have declared that no conflict of interest exists. Submitted: August 4, 2016; Accepted: December 30, 2016.

Reference information: / Clin Invest. 2017;127(4):1338-1352.

https://doi.org/10.1172/JCl89857.
This approach has demonstrated measurable success in treating chronic viral infections, such as hepatitis $\mathrm{C}$ virus (HCV). However, such an approach to drug development is inefficient, expensive, and, therefore, not easily scalable to address the large unmet clinical need (7). Moreover, targeting virally encoded factors by monotherapy often is associated with rapid emergence of drug resistance (7). One alternative approach to treating viral infections while increasing the barrier to resistance is to target host functions, which the viruses intimately rely on (7). Moreover, focusing on host factors commonly required by multiple viral pathogens could provide broad-spectrum coverage. The host-targeted approach is attractive, particularly for the treatment of emerging viral infections lacking any treatment, given the opportunities to repurpose already existing drugs that are known to modulate specific host functions with tolerable side effect and toxicity profiles.

Intracellular membrane traffic is one of many cellular processes hijacked by viruses. Membrane traffic relies, in part, on the interactions between adaptor protein complexes (AP1 through AP5) and the transmembrane cargo. The well-characterized clathrin-associated APs, AP1 and AP2, are heterotetrameric complexes, which orchestrate the formation of vesicles destined for bidirectional transport in the secretory pathway and for endocytosis from the plasma membrane, respectively (8). The 2 host cell kinases AP2-associated protein kinase 1 (AAK1) and cyclin G-associated kinase (GAK) regulate 
A
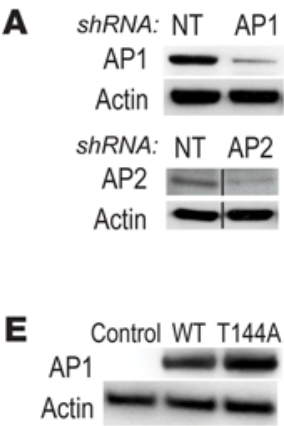

G
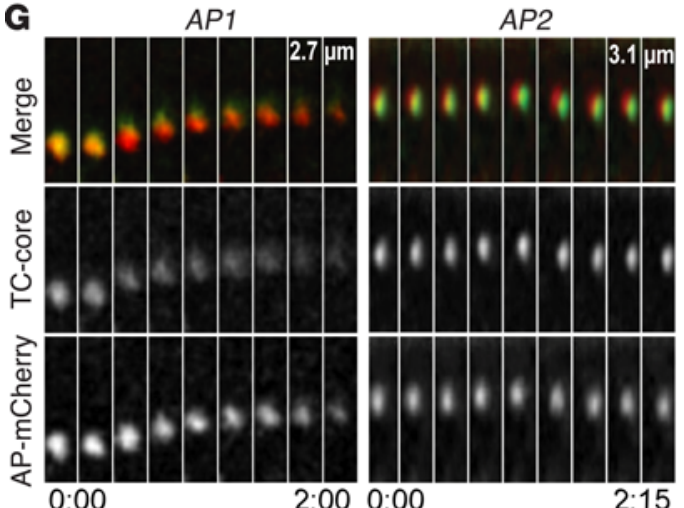

C

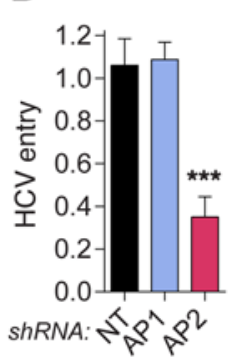

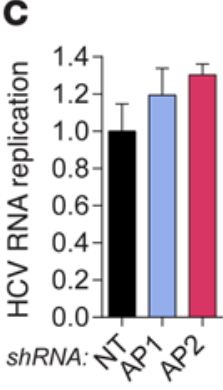

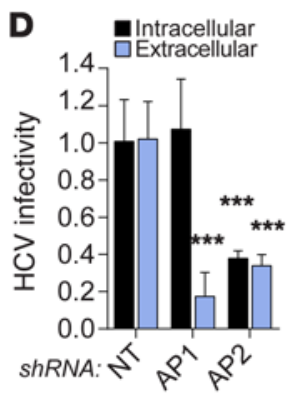

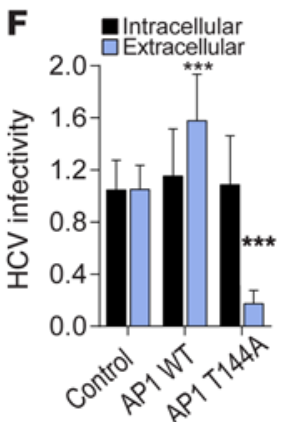

H

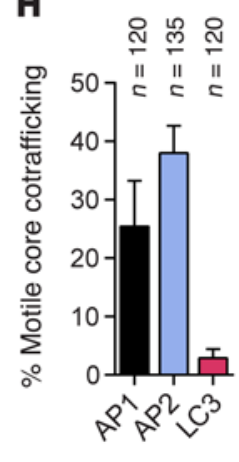

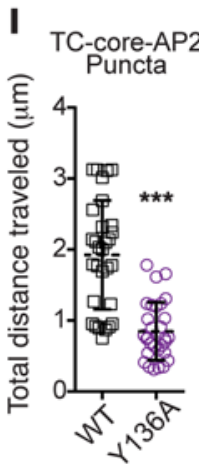
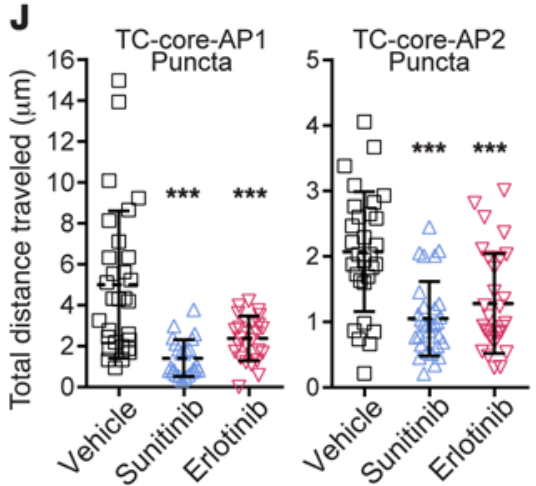

Figure 1. AP1 and AP2 cotraffic with HCV and orchestrate infection. (A) Confirmation of gene expression knockdown by Western blot in Huh7.5 cells stably expressing AP shRNA or nontargeting control (NT). (B) Entry of HCV pseudoparticles (HCVpp) was measured by luciferase assays at 48 hours after infection. (C) HCV RNA replication measured via luciferase assays 72 hours after HCV RNA electroporation. (D) HCV infectivity measured via luciferase assays by inoculation of naive cells with lysates (intracellular) and supernatants (extracellular) from electroporated cells. (E) AP1 ectopic expression following transfection of Huh7.5 cells with GLuc-tagged WT, T144A AP1, or an empty control; blotted with anti-GLuc antibody. (F) HCV intra- and extracellular infectivity in AP1-overexpressing cells versus control. Shown are means \pm SD $(n=3-10)$. (G) Representative live cell fluorescence microscopy montages of TC-core HCV (green) cotrafficking with AP1- and AP2-mCherry (red). Distance traveled ( $\mu \mathrm{m}$ ) and time elapsed (min:s) during video acquisition are indicated. (H) Quantification of motile TC-core puncta cotrafficking with AP1, AP2, and LC3. (I) Quantification of distance traveled per acquisition of WT or Y136A mutant TC-core HCV associated with AP2. (J) Quantification of distance traveled per acquisition of TC-core HCV associated with AP1 or AP2 upon treatment with sunitinib $(4 \mu \mathrm{M})$ and erlotinib $(10 \mu \mathrm{M})$. Results in B-D and $\mathbf{F}$ represent data pooled from at least 2 independent experiments each with $6-10$ biological replicates. $\mathbf{H}-\mathbf{J}$ are representative experiments out of at least 3 conducted. Shown are means \pm SD; ${ }^{* *} P<0.001$ relative to corresponding NT (B-D), empty vector control (F), WT TC-core (I), or vehicle control (J) by 1-way ANOVA, followed by Dunnett's (B, D, and J) or Tukey's (F) multiple comparisons test or 2-tailed unpaired $t$ test (I).

receptor-mediated endocytosis and trans-Golgi network (TGN) transport (9-12). Specifically, AAK1 and GAK phosphorylate the $\mu$ subunits of AP1 and AP2, thereby enhancing their binding affinity for sorting motifs within the cargo $(9,10,13-15)$. Moreover, GAK recruits clathrin-associated APs to the plasma membrane and TGN (16).

Other groups have implicated APs in the life cycle of multiple unrelated viruses (17-26), and we reported roles for AP2 in HCV entry and assembly $(22,23)$. Our work demonstrated that through AP2 phosphorylation, AAK1 and GAK regulate these temporally distinct steps of the HCV life cycle, thereby, for the first time, uncovering their role as "master regulators" of a viral infection $(22,23)$. Moreover, we reported that sunitinib and erlotinib, approved anticancer drugs with potent binding to AAK1 and GAK (dissociation constant $\left[K_{\mathrm{D}}\right]$ of 11 and $3.1 \mathrm{nM}$, respectively; ref. 27), or selective, chemically distinct GAK inhibitors, block HCV entry and assembly in tissue culture $(22,23,28)$. Although clathrin APs are presumed to mediate intracellular viral trafficking, this hypothesis has not been addressed in live cells. Moreover, the roles of AAK1 and GAK in viral infections beyond $\mathrm{HCV}$ and their in vivo functional relevance remain unknown. In the present study, we demonstrate a role for AAK1- and GAK-regulated AP1 activity in HCV release and document that HCV particles specifically cotraffic with AP1 and AP2 in live cells. We also show a requirement for AAK1 and GAK in the life cycles of DENV and EBOV. Furthermore, we provide support for the feasibility of repurposing sunitinib/erlotinib combination as a broad-spectrum antiviral approach using in vitro models of multiple viral infections and murine models of DENV and EBOV. Lastly, we characterize the mechanism of action of sunitinib and erlotinib by validating AAK1 and GAK as critical mediators of the antiviral effect and revealing additional potential antiviral host targets, which include AXL receptor tyrosine kinase (AXL), KIT proto-oncogene receptor tyrosine kinase (KIT), and the proto-oncogene RET.

\section{Results}

APs cotraffic with HCV and orchestrate infection. To determine the differential roles of AP1 and AP2 in HCV infection, we examined the effect of depleting their $\mu$ subunits in Huh7.5 human hepatoma 
A

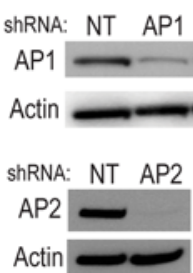

E

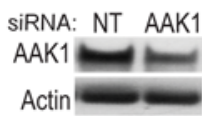

SiRNA: NT GAK

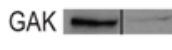

Actin
B

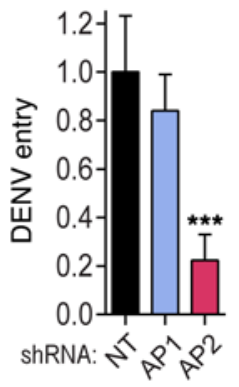

C

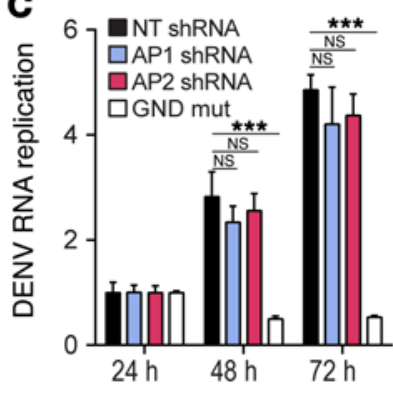

D

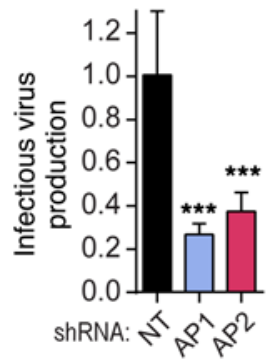

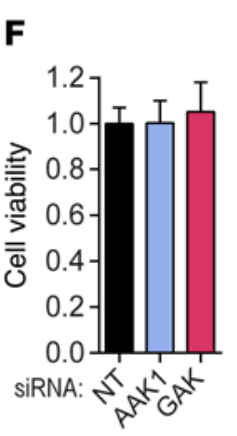
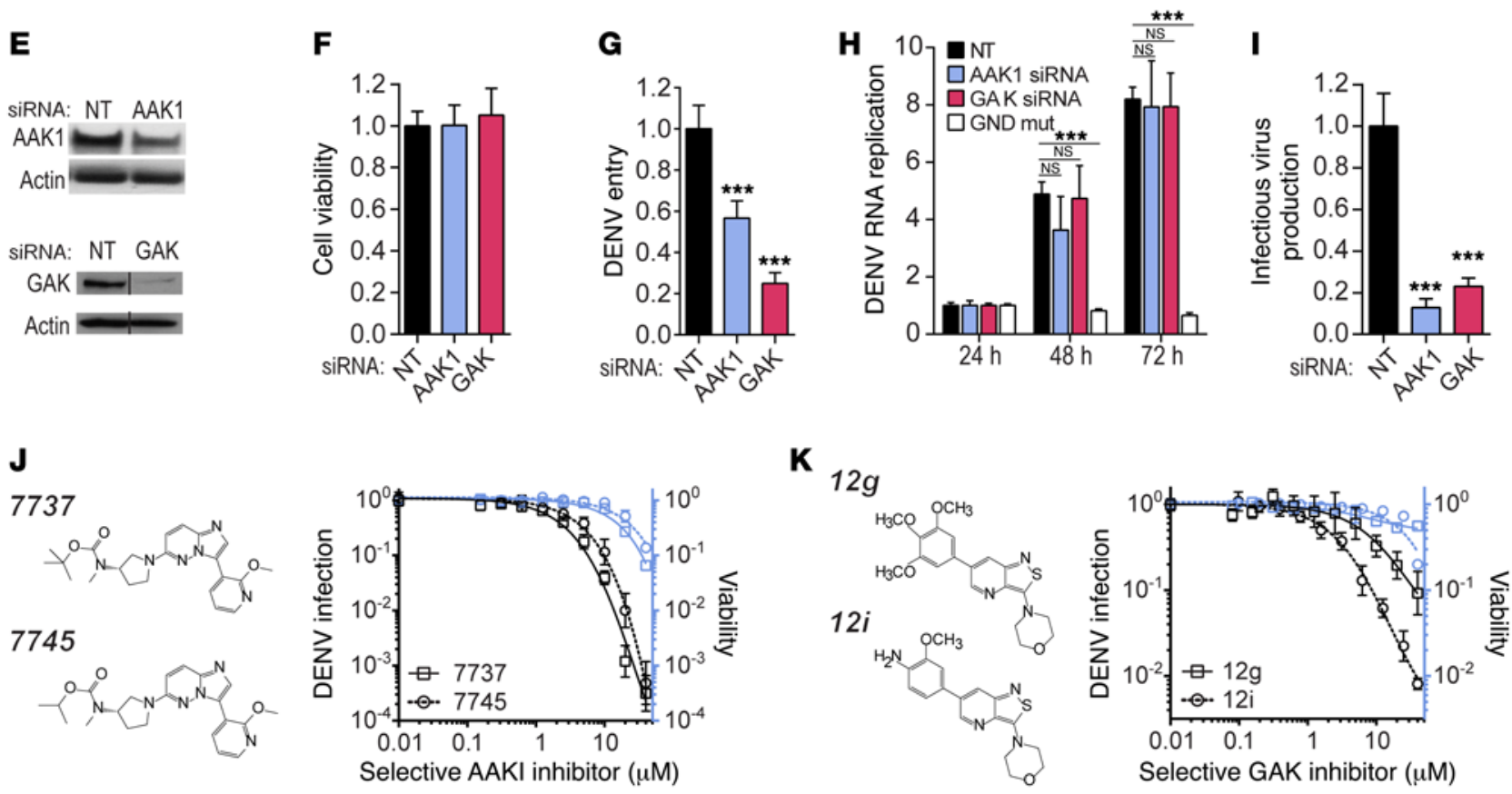

Figure 2. AP1, AP2, and their regulatory kinases, AAK1 and GAK, are essential for DENV infection in vitro. (A and E) Confirmation of stable shRNAmediated (A) or transient siRNA-mediated (E) gene expression silencing by Western blot in Huh7 cells. (B and G) DENV entry measured via luciferase assay 6 hours after infection. ( $C$ and $\mathbf{H})$ DENV RNA replication monitored by luciferase activity every 24 hours following transfection of Huh7 cells with a Tetinducible DNA-launched DENV replicon and induction by doxycycline for 6 hours (GND is a replication-incompetent DENV). Data are normalized to signal at 24 hours. (D and I) Infectious DENV production measured via luciferase assays by inoculation of naive cells with supernatants from stable or siRNAtransfected cells 48 hours after electroporation with DENV RNA. (F) Relative cell viability following gene expression knockdown measured by alamarBlue assays. (J and K) Cell viability (blue) and dose response of DENV infection (black) to more selective AAK1 (J) and CAK (K) inhibitors (structures shown) measured by luciferase assays 48 hours after infection. Data are plotted relative to vehicle control. Shown are representative experiments from at least 2 conducted. Individual experiments in B-D and F-K had 8-10 biological replicates; shown are means \pm SD; ${ }^{* *} P<0.001$ relative to corresponding NT control by 1-way ANOVA (B, D, F, G, and I) or 2-way ANOVA (C and $\mathbf{H})$, followed by Dunnett's multiple comparisons test.

cells on distinct steps of the viral life cycle. Successful depletion of each targeted AP was confirmed (Figure 1A), with no alteration in the expression of the nontargeted AP (data not shown). AP2 depletion reduced HCV entry, had no effect on HCV RNA replication, and reduced HCV assembly (i.e., reduced intra- and extracellular infectivity in lysates and culture supernatants derived from HCVtransfected cells, respectively), as we previously reported (Figure 1, B-D, and refs. 22, 23). In contrast, AP1 depletion reduced extracellular infectivity only (Figure 1, B-D), consistent with a defect in viral release, in agreement with prior reports $(29,30)$. To test whether, in addition to AP2 (22), phosphorylation of AP1 by AAK1 and GAK is important for infectious HCV production, we studied the effect of overexpressing phosphorylation site mutant AP1 (T144A) on HCV infectivity (Figure 1E and ref. 31). Intracel- lular infectivity was not affected by overexpression of either WT or T144A AP1 (Figure 1F). In contrast, extracellular infectivity increased upon ectopic expression of WT AP1 and decreased with ectopic expression of T144A AP1 (Figure 1F). Thus, viral release emerges as yet another step of the HCV life cycle, beyond entry and assembly, which is regulated by AAK1 and GAK.

While APs were postulated to directly mediate intracellular viral trafficking, this has never been addressed experimentally in live cells with any virus. To test the hypothesis that HCV particles shuttle with clathrin APs intracellularly, we used live cell imaging. The cotrafficking of individual, infectious HCV particles harboring a tetracysteine (TC) tag within the core protein (TC-core) with AP1- or AP2-mCherry was monitored (32). We previously have shown that TC-core motility requires HCV virion assembly (32). 
A<smiles>CCN(CC)CCNC(=O)C1C(C)NC(CC2C(=O)NC3CCC(F)CC32)C1C</smiles>

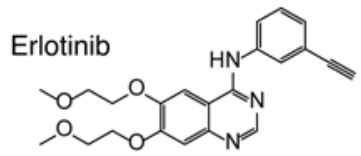

D

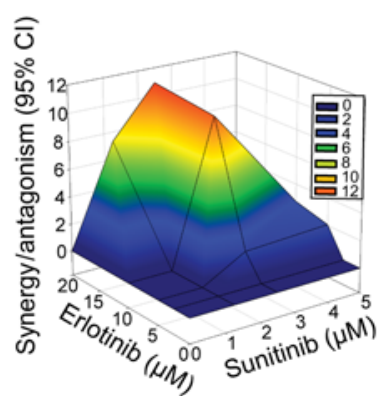

B

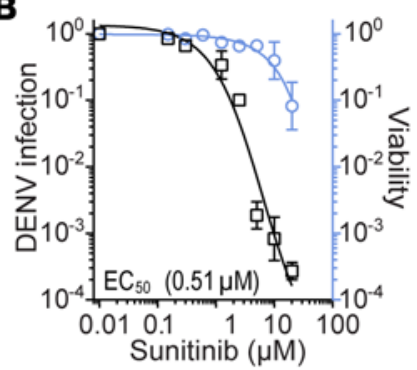

E

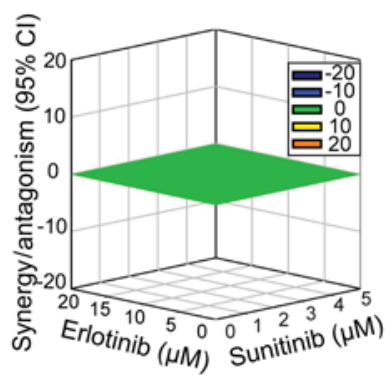

C

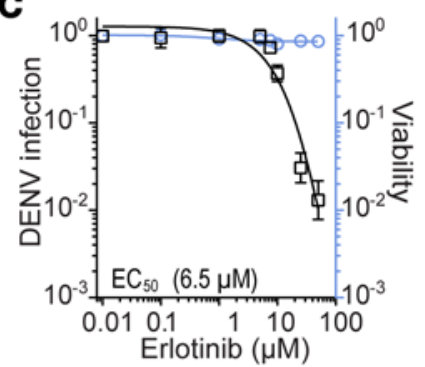

$\mathbf{F}$

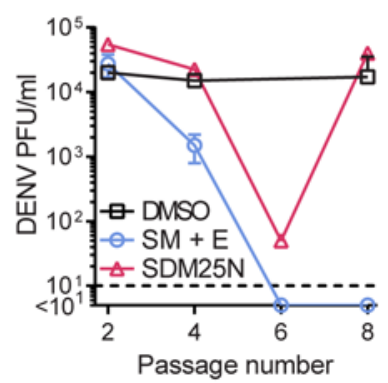

Figure 3. Sunitinib and erlotinib have a synergistic anti-DENV effect and a high genetic barrier to resistance in vitro. (A) Chemical structures of the indicated drugs. (B and C) Cellular viability (blue) and dose response of overall DENV infection (black) to sunitinib and erlotinib measured by luciferase assays at 48 hours after infection. Data are plotted relative to vehicle control. (D and E) Synergy/antagonism at the $95 \% \mathrm{Cl}$ of sunitinib/erlotinib combination treatment on antiviral effect (D) and cellular viability (E) computed by MacSynergy II. (F) DENV4 was used to infect Huh7 cells and passaged every 72 hours by inoculation of naive cells with equal volumes of viral supernatants under DMSO treatment or selection with sunitinib and erlotinib (SM + E) or SDM25N (DENV NS4B inhibitor) increasing from 0.5 to $2.5 \mu \mathrm{M}$ over 8 passages. Viral titers were measured by plaque assays at every other passage. Dashed line represents assay detection limit. Results in $\mathbf{B}$ and $\mathbf{C}$ represent data pooled from at least 2 independent experiments. Data in $\mathbf{D}-\mathbf{F}$ are representative of at least 2 experiments. Shown in B, C, and $\mathbf{F}$ are means \pm SD. Individual experiments in $\mathbf{B}-\mathbf{E}$ and $\mathbf{F}$ had $5-10$ and 2 biological replicates, respectively.

Analysis of TC-core puncta stained with the biarsenical dye FIAsH revealed that a large fraction of motile TC-core cotrafficked with either AP1 (25\%) or AP2 (38\%), whereas only 3\% cotrafficked with the autophagosomal marker LC3 (Figure 1, G and H, Supplemental Figure 1A, and Supplemental Videos 1-3; supplemental material available online with this article; https://oi.org/10.1172/ JCI89857DS1). The velocities of the cotrafficking particles were consistent with previous reports on secretory vesicle trafficking as well as TC-core puncta cotrafficking with vesicle-associated membrane protein (VAMP) (refs. 32, 33, and Supplemental Figure 1B). AP2-associated TC-core puncta motility was reduced significantly upon mutation (Y136A) of a YxxФ motif within core, a motif critical for AP2 binding and HCV assembly (22), as measured by the overall distance traveled (Figure 1I and Supplemental Video 4). These findings provide direct experimental evidence for a role of clathrin-associated APs in mediating intracellular virus trafficking. Specifically, these imaging data combined with our current and previous characterization of the roles of AP1 and AP2 in the life cycle of $\operatorname{HCV}(22,23)$ support the hypothesis that AP1 cotraffics with $\mathrm{HCV}$ during viral release, while AP2 cotraffics with $\mathrm{HCV}$ during viral entry and subsequently to the sites of assembly.

To understand whether drug modulation of AP phosphorylation by AAK1 and GAK manifests itself in an intracellular trafficking defect, we studied the effect of sunitinib and erlotinib, approved drugs with potent anti-AAK1 and/or anti-GAK activity, on HCV particle trafficking by live cell imaging. Treatment of HCV-infected cells with sunitinib and erlotinib reduced motility of TC-core puncta cotrafficking with AP1 and AP2 (Figure 1J and
Supplemental Videos 5-10). These findings support our hypothesis that the antiviral effect of sunitinib and erlotinib is associated with reduced intracellular viral traffic.

The role of $A A K 1$ and GAK in DENV infection in cells. The requirement for AAK1 and GAK in viral infections beyond HCV is unknown. To investigate whether another, distantly related member of the Flaviviridae family relies on these regulatory kinases and their associated AP targets, we examined the effect of the corresponding gene silencing on DENV infection in human hepatoma (Huh7) cells. We observed a requirement for AP2, but not AP1, in DENV entry using cell lines stably expressing shRNA targeting AP1, AP2, or a nontargeting (NT) sequence (Figure 2, A and B). While depletion of AP1 and AP2 had no effect on DENV RNA replication as measured by subgenomic replicon assays (ref. 34 and Figure 2C), it diminished the production of infectious virus in culture supernatants (Figure 2D). Silencing expression of AAK1 and GAK resulted in no apparent cytotoxic effect (Figure 2, E and F) but, analogously to experiments with HCV, inhibited entry and infectious virus production of DENV, with no effect on RNA replication (Figure 2, G-I). These results implicate AAK1 and GAK in the DENV life cycle via regulation of 2 temporally distinct steps that depend on the clathrin-associated APs: entry and infectious virus production.

To determine whether a comparable effect on DENV infection can be achieved pharmacologically and further validate AAK1 and GAK as antiviral targets, we treated DENV-infected cells with selective AAK1 and GAK inhibitors. The imidazo[1,2-b]pyridazine-based compounds 7737 and 7745 were originally developed 
Table 1. Antiviral activity of sunitinib and erlotinib

\begin{tabular}{|c|c|c|c|c|c|c|}
\hline Family & Virus & Strain & Sunitinib $\mathrm{EC}_{50} / \mathrm{CC}_{50}(\mu \mathrm{M})$ & Erlotinib $\mathrm{EC}_{50} / \mathrm{CC}_{50}(\mu \mathrm{M})$ & Cells & Assay \\
\hline \multirow[t]{7}{*}{ Flaviviridae } & $\mathrm{HCV}$ & $\mathrm{J6} / \mathrm{JFH}$ & $1.2 />10$ & $0.6 />15$ & Huh7.5 & Luciferase, FFA \\
\hline & DENV1 & 276RKI, PRS41393 & $0.6 />10$ & $1.9 />20$ & BHK-21 & Plaque \\
\hline & DENV2 & $\begin{array}{l}\text { TSV01, 429557, } \\
\text { New Guinea C }\end{array}$ & $0.51 / 11.5$ & $2.5 />20-6.5 />50$ & Huh7, BНК-21 & Luciferase, plaque \\
\hline & DENV3 & Philippines/H87/1956 & $0.3 />10$ & $1.3 />20$ & BHK-21 & Plaque \\
\hline & DENV4 & BC287/97, H241 & $0.23 />10$ & $3.9 />20$ & BHK-21 & Plaque \\
\hline & WNV & NY 993000.0259 & $0.55 />20$ & NE & MEF, Vero & FFA \\
\hline & ZIKV & MR766 & $0.51 / 14.1$ & $6.28 />30$ & Huh7 & Plaque \\
\hline Filoviridae & EBOV & Zaire & $0.47 />10$ & $\begin{array}{l}12.9 />30 \\
2.88 / 15\end{array}$ & $\begin{array}{l}\text { Huh7 } \\
\text { Vero }\end{array}$ & Immunostaining \\
\hline Togaviridae & CHIKV & $181 / 25$ & $4.67 / 11.9$ & $0.7 />30$ & Vero & Plaque \\
\hline Arenaviridae & JUNV & Candid 1 & $4.8 / 10.4$ & $1.7 />20$ & Vero & CPE \\
\hline Retroviridae & HIV & NL4-3 & $0.8 />20$ & $2 />20$ & HeLa/TZM-b1 & Luciferase \\
\hline Paramyxoviridae & RSV & $\mathrm{A} 2$ & $<0.12 / 12.5$ & $<0.12 />30$ & Нер2 & Immunoplaque \\
\hline
\end{tabular}

$\mathrm{EC}_{50}$, half-maximal effective concentration; $\mathrm{CC}_{50}$, half-maximal cellular cytotoxicity; FFA, focus-forming assay; NE, no effect; $C P E$, cytopathic effect. Virus

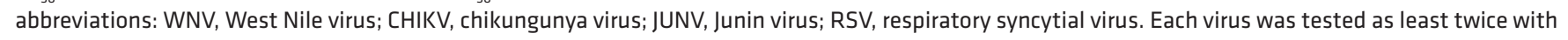
3 or more technical replicates.

to modulate AAK1 activity as a potential treatment of neurological disorders $\left(K_{\mathrm{D}}=1 \mathrm{nM}, \mathrm{IC}_{50}<10 \mathrm{nM}\right.$ ) (Figure 2J, Supplemental Figure 2, and ref. 35). The isothiazolo[5,4-b]pyridines $12 \mathrm{~g}$ and $12 \mathrm{i}$ (Figure $2 \mathrm{~K}$ ) are potent $\left(K_{\mathrm{D}}=\sim 8 \mathrm{nM}\right)$, selective, ATP-competitive GAK inhibitors capable of restricting HCV infection (28). We measured a dose-dependent inhibition of the DENV serotype 2 (DENV2) infection following a 2-day drug treatment with all 4 compounds, with half-maximal effective concentrations $\left(\mathrm{EC}_{50} \mathrm{~s}\right)$ of 1.5-5.1 $\mu \mathrm{M}$ (Figure 2, J and $\mathrm{K}$ ). The concentration range yielding at least $1 \log$ reduction in viral infection showed minimal or no toxicity as measured by alamarBlue assays.

Together, these results validate AAK1 and GAK as regulators of DENV infection and point to their pharmacological inhibition as a potential anti-DENV strategy.

Sunitinib and erlotinib have a synergistic anti-DENV effect and a high genetic barrier to resistance in vitro. To determine whether a similar effect on DENV infection can be achieved with approved drugs with potent anti-AAK1 and/or anti-GAK activity, we treated DENV-infected cells with sunitinib and erlotinib (Figure 3A). Consistent with published HCV data $(22,23)$, we measured a dose-dependent inhibition of DENV2 infection following a 2-day drug treatment with an $\mathrm{EC}_{50}$ of 0.51 $\mu \mathrm{M}$ for sunitinib and $6.5 \mu \mathrm{M}$ for erlotinib by luciferase assays (Figure 3, B and C). The concentration range yielding at least 2 log reduction in viral infection showed minimal or no toxicity as measured by alamarBlue assays with half-maximal cellular cytotoxicities $\left(\mathrm{CC}_{50} \mathrm{~s}\right)$ of $8.0 \mu \mathrm{M}$ for sunitinib and $>50 \mu \mathrm{M}$ for erlotinib (Figure 3, B and C, and Table 1). Similar results were demonstrated by standard plaque assays (Supplemental Figure $3 \mathrm{~A})$. Notably, treatment with combinations of the 2 drugs revealed synergistic inhibition of DENV2 infection with a synergy volume of $36.7 \mu \mathrm{M}^{2} \%$ at the $95 \%$ CI and no synergistic toxicity (Figure 3, D and E, and Supplemental Figure 3B). Importantly, sunitinib and erlotinib also dose-dependently inhibited infection of DENV1, DENV3, and DENV4 (Table 1).
To determine whether DENV can escape treatment with sunitinib and erlotinib, we passaged DENV in the presence of sunitinib/erlotinib combination or the DENV nonstructural (NS) $4 \mathrm{~B}$ protein inhibitor SDM25N at increasing concentrations (0.5-2.5 $\mu \mathrm{M})$ corresponding to values between $\mathrm{EC}_{50}$ and $\mathrm{EC}_{90}$. Infectious virus output was quantified over several passages by plaque assays. By passage 8, DENV4 overcame inhibition by SDM25N with the emergence of a previously characterized resistance mutation in NS4B (P101L, analogous to P104L in DENV2) (36). In contrast, DENV4 was cleared from the culture by passage 6 under the sunitinib/erlotinib treatment without any phenotypic resistance (Figure 3F). These results point to sunitinib/erlotinib combination as a potential anti-DENV strategy with a higher relative barrier to resistance than a direct-acting antiviral.

Broad-spectrum activity of sunitinib and erlotinib. Next, we studied the effect of sunitinib and erlotinib on replication of 2 additional flaviviruses, West Nile virus (WNV) and ZIKV. Sunitinib dose-dependently inhibited both WNV and ZIKV by focusformation and plaque assays with $\mathrm{EC}_{50}$ of $0.51-0.55 \mu \mathrm{M}$, whereas erlotinib demonstrated some efficacy only against ZIKV with $\mathrm{EC}_{50}$ of 6.28 $\mu \mathrm{M}$ (Supplemental Figure 4, A and B, and Table 1).

We also investigated whether EBOV, a member of an unrelated viral family (Filoviridae), whose entry depends on AP1 and AP2 activity (18, 37-39), may be similarly dependent on AAK1 and GAK. To test this hypothesis, we silenced AAK1 and GAK expression in Vero cells (Figure 4A) and measured infection of vesicular stomatitis virus encapsidated RNA (encoding a GFP reporter gene) pseudotyped with EBOV glycoproteins (rVSV-GP EBOV). Quantification of GFP-positive cells at the 20-hour time point by flow cytometry revealed that AAK1 and GAK depletion reduced infection relative to NT control (Figure 4C) without impacting cell viability (Figure 4B). A similar level of inhibition was observed upon quantification of viral RNA at 3 hours after infection, highlighting a defect in the entry step (Supplemental Figure 5A). These data further validate AAK1 and GAK as targets for broad-spectrum antiviral therapy. More- 
A

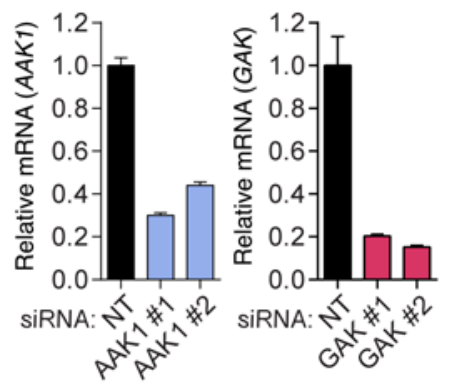

E

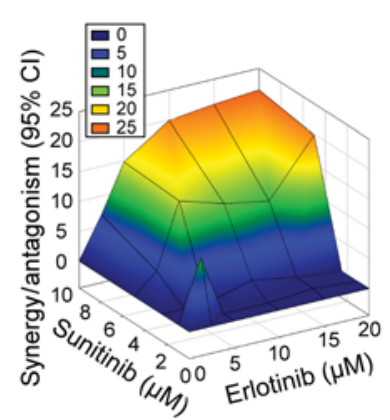

B

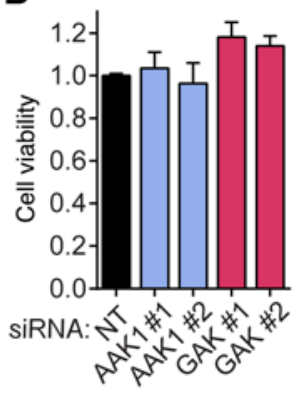

$\mathbf{F}$

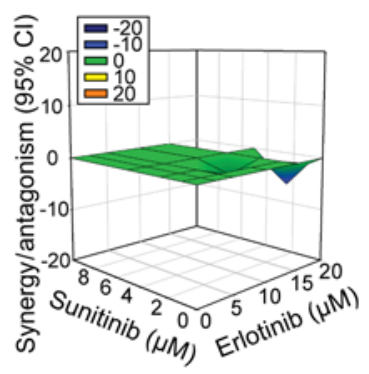

C

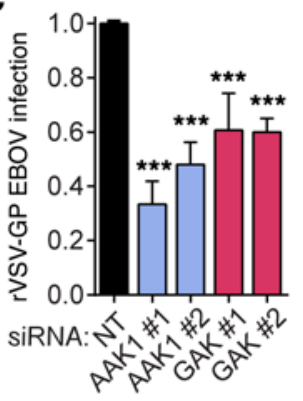

G

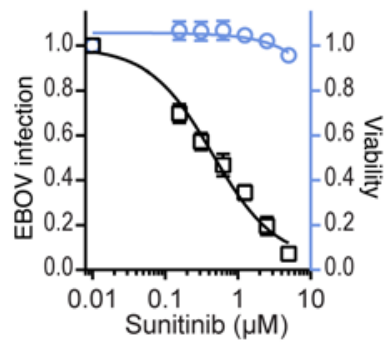

D
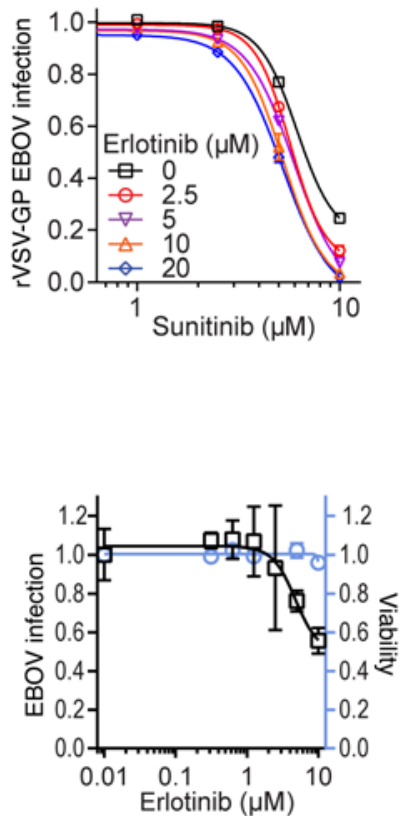

Figure 4. Inhibition of AAK1 and GAK suppresses EBOV infection. (A) Confirmation of siRNA-mediated gene expression silencing by quantitative PCR in Vero cells. Shown is normalized gene expression relative to GAPDH at 48 hours after transfection. (B and C) Relative cell viability measured by alamarBlue assays (B) and rVSV-GP EBOV infection of Vero cells measured by flow cytometry (C) at 20 hours after infection of AAK1- and GAK-depleted cells. ${ }^{* *} P<0.001$ relative to NT (1-way ANOVA followed by Dunnett's multiple comparisons test). (D) Dose response of rVSV-GP EBOV infection to 4-hour treatment with inhibitors measured by flow cytometry at 20 hours after infection in Vero cells. (E and $\mathbf{F}$ ) Synergy/antagonism of sunitinib/ erlotinib combination treatment on rVSV-GP EBOV infection (E) and cell viability (F). (C) Dose response to drug treatment in Huh7 cells under biosafety level 4 containment following 48 hours with EBOV infection (black) measured by immunostaining with an anti-GP antibody, and cell viability (blue) measured by Hoechst counterstain and quantified by a high-content imager. Data are plotted relative to vehicle control. All data shown are representative of at least 2 experiments; B-G have 3 biological replicates each. Shown in B-D and $\mathbf{G}$ are means \pm SD.

over, treatment of Vero cells with sunitinib and erlotinib resulted in a dose-dependent decrease in rVSV-GP EBOV infection measured by flow cytometry 20 hours after infection and entry measured by quantitative reverse transcriptase PCR (qRT-PCR) 3 hours after infection (Figure 4D and Supplemental Figure 5B). Although the effect of erlotinib measured by flow cytometry was modest relative to that of sunitinib, combination treatment displayed measurable synergy in inhibiting entry with a synergy volume of $147.05 \mu \mathrm{M}^{2} \%$ at the 95\% CI and zero synergistic toxicity (Figure 4, E and F). Next, we tested the ability of these drugs to inhibit authentic EBOV infection in Huh7 cells. Sunitinib treatment resulted in a dose-dependent reduction in EBOV infection with $\mathrm{EC}_{50}$ value of $0.47 \mu \mathrm{M}$ and $\mathrm{CC}_{50}$ greater than $10 \mu \mathrm{M}$, whereas erlotinib showed moderate activity with $\mathrm{EC}_{50}$ of $12.9 \mu \mathrm{M}$ and no appreciable cytotoxicity at the concentrations tested (Figure $4 \mathrm{G}$ and Table 1 ).

To explore more broadly the spectrum of coverage provided by sunitinib and erlotinib, we studied their antiviral effects against additional unrelated viruses. Viral infection was measured in various cell lines following 3-day treatment regimens. We detected antiviral activity of either or both drugs against RNA viruses in 6 families (Table 1), including Togaviridae (e.g., chikungunya virus [CHIKV]), Arenaviridae (e.g., Junin virus [JUNV]), and Paramyxoviridae (e.g., respiratory syncytial virus [RSV]). These data expand the possible indications of sunitinib and/or erlotinib as antiviral agents beyond Flaviviridae infections, to other established and emerging RNA viruses.
Sunitinib/erlotinib combinations are effective in vivo. To address the therapeutic potential of sunitinib and erlotinib as antiviral agents, we tested their application in a murine model of dengue. We measured viral burden and mortality in an IFN$\alpha / \beta$ and IFN- $\gamma$ receptor-deficient murine model of dengue on 129/Sv (AG-129) (40, 41) and C57BL/6 (AG-B6) genetic backgrounds. In a prophylaxis model, we initiated once-daily treatment of AG-B6 mice with $30-60 \mathrm{mg} / \mathrm{kg}$ of sunitinib and erlotinib in combination or individually concurrently with DENV inoculation and analyzed viremia at 48 hours. The doses tested for each drug were at or near the equivalent of approved human dose as calculated based on the body surface area per the FDA's guidelines (42). These doses were below the maximum tolerated dose (MTD) in mice and confirmed to be nontoxic in our dengue model (42-44). Treatment with erlotinib did not alter viremia, whereas sunitinib alone marginally reduced it (Figure $5 \mathrm{~A})$. Consistent with our in vitro synergy results, daily administration of the combination treatment resulted in 11-fold reduction in viral RNA (Figure $5 \mathrm{~A}$ ). In addition, we measured a significant reduction of the infectious virus load by plaque assays in the serum as well as spleen and liver in mice treated with $30 \mathrm{mg} / \mathrm{kg}$ doses of sunitinib and erlotinib relative to vehicle controls (Supplemental Figure 6A). Pharmacokinetic analysis revealed that within the first 6 hours of administration each drug concentration in the serum, as measured by liquid chromatography-tandem mass spectrometry, exceeded the 
A

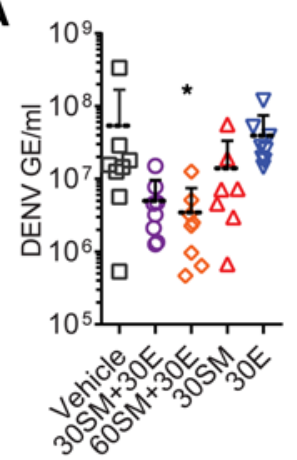

D

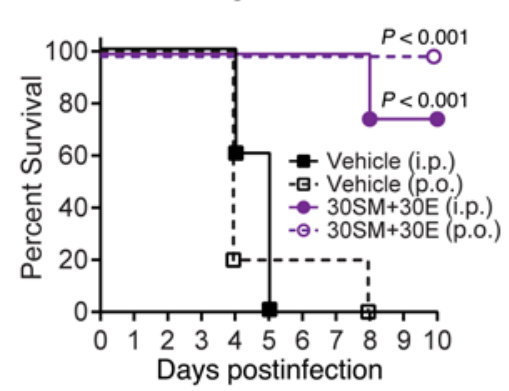

G

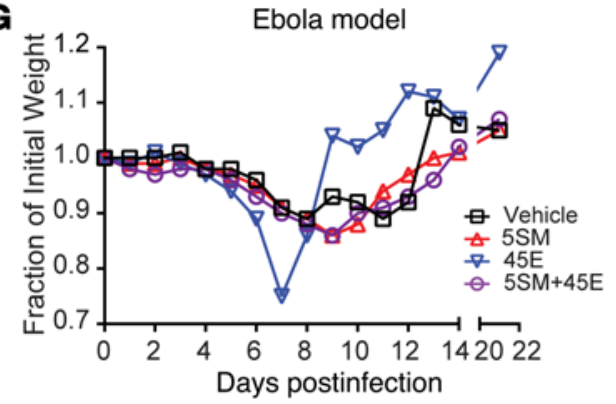

B

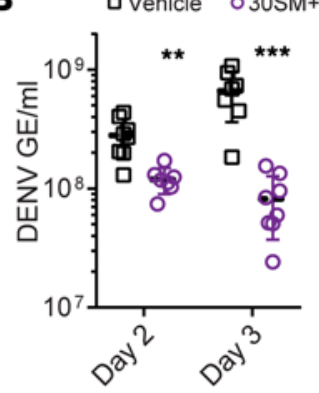

C

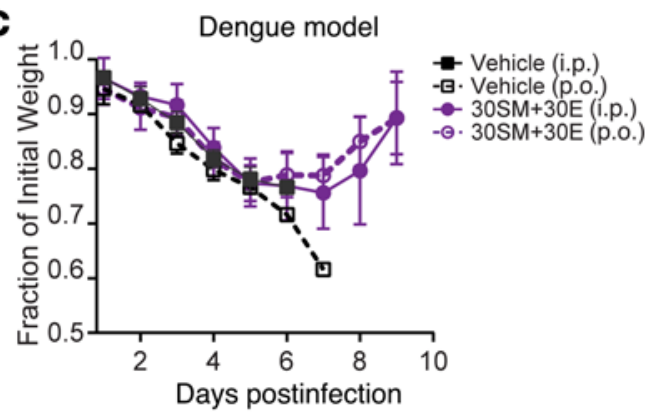

E

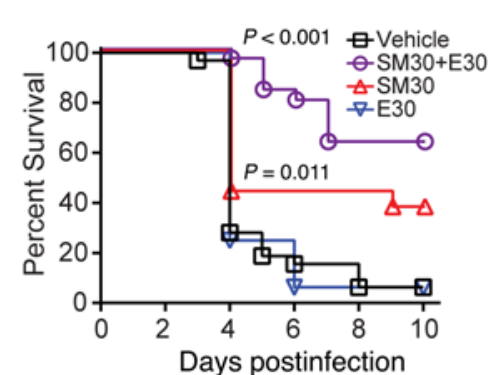

$\mathbf{F}$

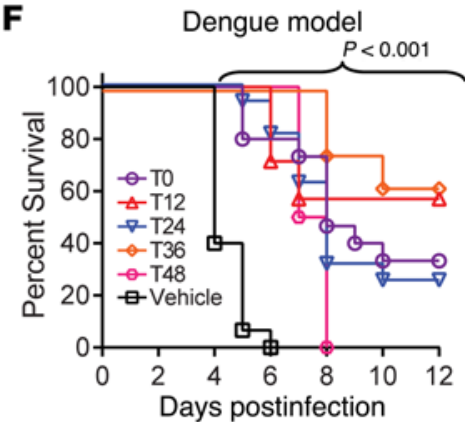

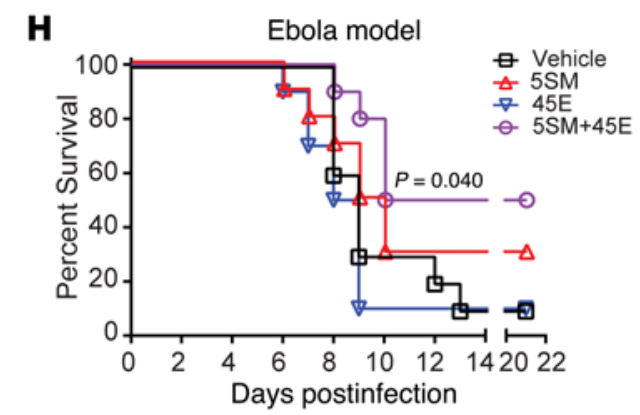

Figure 5. AAK1 and GAK inhibitors are protective in murine models of dengue and Ebola. (A) DENV viremia in AG-B6 mice measured by qRT-PCR on day 2 postinfection following once-daily administration of vehicle, sunitinib (SM), and/or erlotinib (E). (B) DENV viremia in AG-B6 mice on days 2 and 3 postinfection following twice-daily drug administration. (C and D) Weight loss (C) and mortality (D) of DENV-infected AG-B6 mice treated once daily for 5 days with vehicle or sunitinib/erlotinib combination ( $n=8$ per treatment group). (E) Mortality of DENV-infected AG-129 mice treated once daily for 5 days with vehicle, sunitinib, and/or erlotinib (data are pooled from 2 independent experiments, $n=8-16$ per treatment group). (F) Mortality of DENV-infected AG-B6 mice treated once daily with vehicle or sunitinib/erlotinib combination beginning at the indicated hour after inoculation, TOT48 (data are pooled from 2 independent experiments, $n=8-16$ per treatment group). (G and $\mathbf{H})$ Weight loss (C) and mortality (H) of EBOV-infected C57BL/6 mice treated once daily for 10 days with vehicle, sunitinib, and/or erlotinib ( $n=10$ per treatment group). Doses are in $\mathrm{mg} / \mathrm{kg}$. Administration was i.p., except when denoted p.o. (C and $\mathbf{D})$, at inoculation $(\mathbf{A}-\mathbf{E}, \mathbf{G}$, and $\mathbf{H})$ or after inoculation $(\mathbf{F})$. $\mathbf{A}-\mathbf{D}, \mathbf{G}$, and $\mathbf{H}$ are representative of 2 or more independent experiments. ${ }^{*} P<0.05,{ }^{* *} P<0.01,{ }^{* *} P<0.001$ relative to vehicle control by nonparametric Kruskal-Wallis test with Dunn's multiple comparisons post-test $(\mathbf{A})$ or nonparametric Mann-Whitney test (B). Survival analysis (D-F and $\mathbf{H})$ was done with log-rank (Mantel-Cox) test; $P$ values are relative to vehicle control. GE, genomic equivalents.

corresponding $\mathrm{EC}_{50}$ concentration deduced from our in vitro data (Supplemental Figure 6B). Moreover, the synergy between sunitinib and erlotinib predicts even more potency in combination. Although sunitinib and erlotinib were largely cleared from the serum by 18 hours, which is in contrast with the slower clearance rates reported in humans $(44,45)$, both drugs concentrate severalfold within tissues where DENV replicates, such as liver $(46,47)$. To maintain higher serum drug concentration, we next administered $30 \mathrm{mg} / \mathrm{kg}$ drug combination at 12 -hour intervals and measured viremia. The twice-daily drug administration resulted in an even more apparent reduction of viral load rela- tive to vehicle control (Figure 5B). Notably, drug administration reduced viral load from day 2 to 3 postinfection in contrast to an increase in viral load within the control arm during that time.

To assess whether the reduction in viral load would translate into improved disease outcome, we determined the effect of combination treatment with sunitinib and erlotinib on morbidity and mortality in AG-129 and AG-B6 mice. Following infection with a lethal DENV inoculum, we initiated once-daily drug administration at a dose sufficient to significantly reduce viremia (i.e., 30 $\mathrm{mg} / \mathrm{kg}$ of each drug). The animals were monitored twice daily and were euthanized when moribund (48). The experiment was 
A

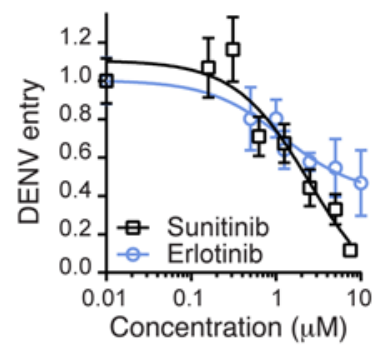

B

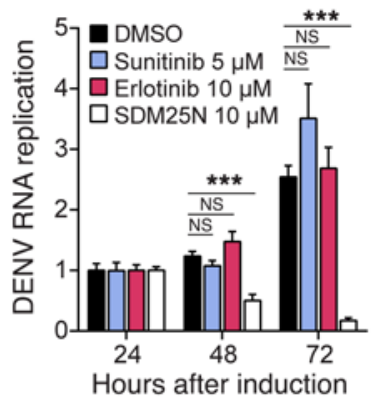

C

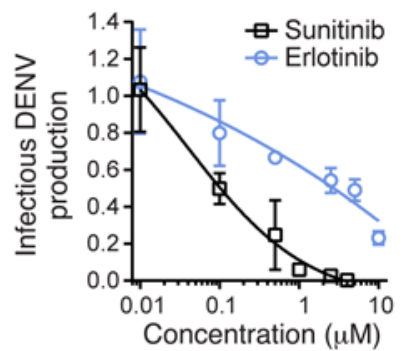

D

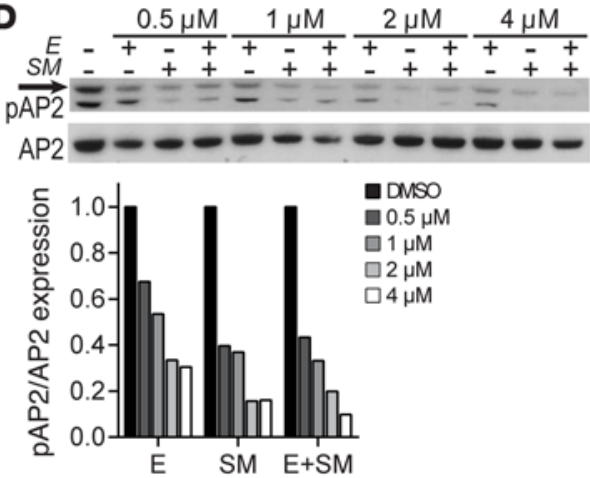

E

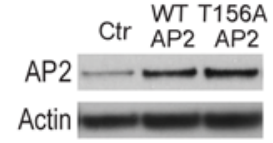

$\mathbf{F}$

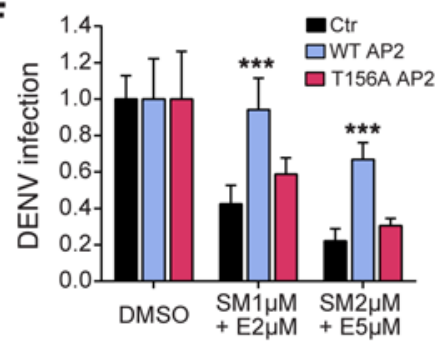

G
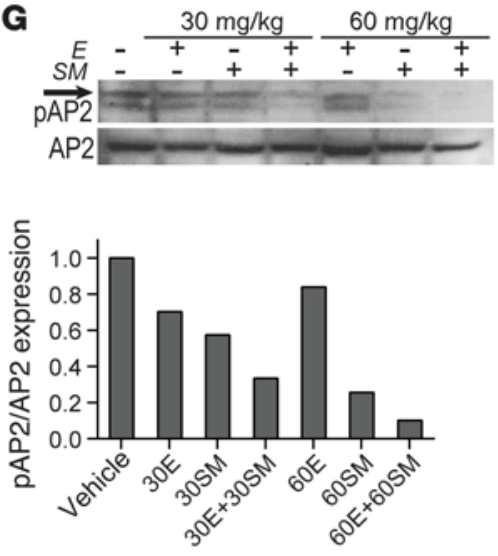

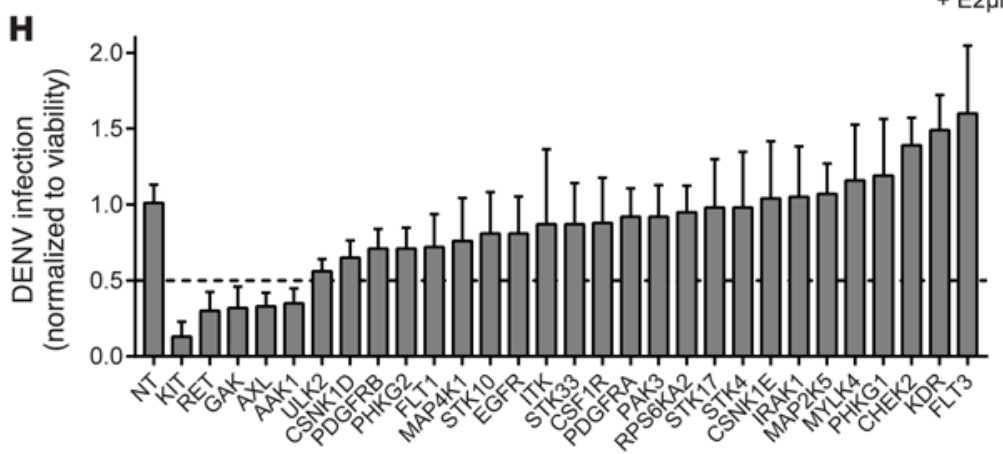

Figure 6. Mechanisms underlying the antiviral effect of sunitinib and erlotinib in vitro and in vivo. (A-C) Huh7 cells were treated with the inhibitors and monitored for DENV entry (A) at 6 hours after infection, DENV RNA replication (B) after induction of replication of DNA-launched DENV replicon, and infectious virus production (C) at 48 hours after electroporation with DENV RNA. SDM25N is an inhibitor of DENV RNA replication. (D) Effect of 1-hour treatment with erlotinib (E) and/or sunitinib (SM) on phosphorylation of AP2 in DENV-infected Huh7 cells measured by Western blotting. Arrow indicates approximately $50 \mathrm{kDa}$. The ratio of phospho-AP2 (pAP2) to total AP2 was quantified. (E) Level of AP2 and actin expression measured by Western blot following lentiviral transduction with control or AP2-expressing constructs. (F) Rescue of DENV infection in the presence of inhibitors upon overexpression of WT or T156A AP2 versus vector control measured by luciferase assays 48 hours after infection. Micromolar concentration of each inhibitor is noted on the $x$ axis. (G) Effect of 3-hour i.p. treatment with erlotinib (E) and/or sunitinib (SM) on phosphorylation of AP2 in liver tissue of AG-B6 mice measured by Western blotting and quantified as the ratio of pAP2 to total AP2. (H) DENV infection relative to NT control following siRNA-mediated knockdown of kinases targeted by sunitinib and erlotinib measured by luciferase assays at 48 hours and normalized to cell viability. Data in A, C, and I are pooled from 2 independent experiments with 4-8 replicates each. Data in the other panels are representative of 2 or more independent experiments. B and $\mathbf{F}$ have at least 5 replicates each. ${ }^{* *} P<0.001$ relative to DMSO by 2-way ANOVA followed by Dunnett's multiple comparisons test (B) or relative to vector control by 1-way ANOVA followed by Dunnett's multiple comparisons test (F).

concluded when all the remaining animals regained full mobility and displayed weight gain for at least 2 consecutive days. Upon a 5-day drug treatment regimen given either i.p. or orally, we observed a significant reduction in morbidity and mortality of infected animals relative to vehicle controls (Figure 5, C and D). Specifically, $100 \%$ of vehicle-treated mice succumbed to infection on day 4-8 postinfection, whereas sunitinib/erlotinib treatment protected $75 \%-100 \%$ of the mice. This combination treatment proved efficacious with either i.p. or oral administration, the latter of which is approved for use in humans (Figure 5, C and D). We also compared the effect of daily treatment with the individual drugs with that of the combination. In this trial, $94 \%$ of vehicle-treated AG-129 mice succumbed to infection; treatment with erlotinib did not alter survival, whereas sunitinib alone offered partial $(37 \%)$ protection. Consistent with our in vitro synergy results, we observed the greatest protection (62\%) from 


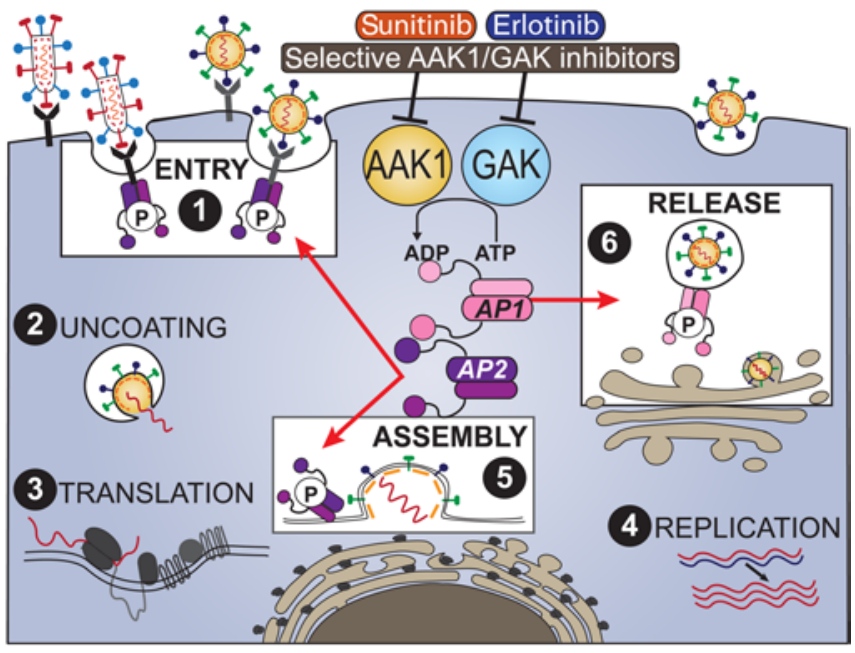

Figure 7. Model: AAK1 and GAK in infection and as broad-spectrum antiviral targets. Host kinases AAK1 and CAK regulate entry, assembly, and/or release of multiple RNA viruses through phosphorylation of the membrane trafficking adaptors AP1 (pink) and AP2 (purple). Sunitinib, erlotinib, and selective inhibitors of AAK1 and GAK disrupt these temporally distinct steps of the viral life cycle and act as broad-spectrum antivirals.

mortality with combination drug treatment (Figure 5E). Furthermore, even when the combination drug treatment was initiated at various time points after infection, the mice remained protected relative to vehicle control, albeit mice treated at 48 hours after inoculation eventually succumbed to infection (Figure $5 \mathrm{~F}$ ).

Given the observed potency of sunitinib and sunitinib/erlotinib combination against EBOV infection in vitro, we assessed in vivo efficacy of these drugs in a murine model of Ebola. Drugs were administered daily i.p. for 10 days beginning at 6 hours before infection. As with the dengue model, the chosen doses were at or near the equivalent of approved human dose, below the MTD in mice, and confirmed to be nontoxic in our Ebola model. All mice demonstrated signs of morbidity as evident by weight loss during the first 7-10 days after infection (Figure $5 \mathrm{G}$ ). Ninety percent of vehicle-treated mice succumbed to infection on days 6-13 postinfection; treatment with erlotinib at $45 \mathrm{mg} / \mathrm{kg}$ did not alter animal survival, whereas $5 \mathrm{mg} /$ $\mathrm{kg}$ sunitinib alone increased survival to $30 \%$ (Figure $5 \mathrm{H}$ ). Consistent with our in vitro synergy results, we observed the greatest weight gain and survival (50\%) with a combination drug treatment.

Together, these results demonstrate therapeutic potential of sunitinib/erlotinib combinations against infections with 2 unrelated emerging RNA viruses.

Mechanisms underlying the antiviral effects of sunitinib and erlotinib in vitro and in vivo. To better understand the target(s) and mechanism of action underlying the anti-DENV activity of sunitinib and erlotinib, we first probed the steps of the viral life cycle affected by these compounds. We detected interference precisely with the steps inhibited via siRNAs against AAK1 and GAK (Figure 2, G and I), namely entry and infectious DENV production (Figure 6, A-C). Notably, DENV RNA replication, a step commonly inhibited by direct-acting antivirals (7), was not affected by these drugs. This phenotype supports a hypothesis that inhibition of AAK1 and GAK likely contributes to the anti-DENV effect of these drugs.
To confirm that the antiviral activity is correlated with functional inhibition of AAK1 and GAK activity, we measured levels of phospho-AP2 upon drug treatment. Dose-dependent reduction in the phospho-AP2 to total AP2 ratio was demonstrated in DENVinfected cells (Figure 6D).

Next, we conducted gain-of-function assays to further validate AP2, a substrate for both AAK1 and GAK, as a key mediator of the anti-DENV effect of these drugs. Ectopic expression of WT but not T156A phosphorylation AP2 mutant or vector control either partially or completely rescued the antiviral effect of sunitinib/erlotinib combination (Figure 6, E and F). These results indicate that AAK1and GAK-mediated phosphorylation of AP2 is a mechanism underlying the antiviral effect of sunitinib and erlotinib against DENV.

Next, we determined whether these drugs exert their antiviral effect in vivo by similarly inhibiting phosphorylation of the AAK1 and GAK ligand AP2. Liver tissue lysates harvested from AG-B6 mice 3 hours after drug administration revealed dose-dependent inhibition of AP2 phosphorylation upon treatment with sunitinib and erlotinib, and most markedly with the combination (Figure $6 \mathrm{G})$. These results provide evidence that drug exposure in animals is associated with modulation of AP2.

These data, combined with the finding that more selective AAK1 and GAK inhibitors lacking affinity to most of sunitinib's and erlotinib's cancer targets (e.g., VEGFR and EGFR) (28, 35) have anti-DENV activity, indicate that AAK1 and GAK are important mediators of the observed antiviral effect. Nevertheless, these data cannot rule out additional potential cellular targets mediating the anti-DENV activity of these compounds. Whereas erlotinib's target selectivity is quite narrowly focused on EGFR and GAK with significantly less affinity for other kinases, sunitinib is a multitarget kinase inhibitor $(49,50)$. We thus examined the effects of siRNA-mediated depletion of 27 major kinases targeted by these small molecules $\left(K_{\mathrm{D}}<20 \mathrm{nM}\right)$ on DENV infection and cellular viability. Using a cutoff of greater than $50 \%$ inhibition of viral infection as measured by luciferase assays normalized to cell viability in 2 independent screens, we identified AXL, KIT, and RET as possible antiviral targets of our kinase inhibitors in addition to AAK1 and GAK (Figure 6H). However, silencing of KIT also substantially reduced cellular viability (Supplemental Figure 7).

Taken together, our data indicate that inhibition of AP-mediated intracellular membrane trafficking regulated by AAK1 and GAK represents an important mechanism by which sunitinib and erlotinib inhibit DENV infection in vitro and in vivo and that additional mechanisms, potentially mediated by other kinases, may act in concert.

\section{Discussion}

Clathrin-associated AP1 and AP2 complexes have been implicated in orchestrating multiple viral infections; however, their precise mechanistic involvement was not characterized. Moreover, the relevance of AAK1 and GAK, kinase regulators of these APs that we discovered as essential for HCV infection, to other viral infections remained unknown. Here, we addressed this knowledge gap and evaluated the therapeutic potential of inhibiting AAK1 and GAK as a broad-spectrum antiviral strategy. Integrating RNAi, dominant interfering, pharmacological and molecular virology 
approaches, we demonstrate roles for AP1 and AP2 complexes as well as AAK1 and GAK in entry and assembly/release of Flaviviridae family members and validate these host factors as attractive targets for broad-spectrum antiviral therapy (Figure 7). We establish that sunitinib and erlotinib inhibit DENV and EBOV infections in vitro and in vivo, and are potent in vitro against WNV, ZIKV, and RNA viruses from 4 additional families: Togaviridae, Arenaviridae, Paramyxoviridae, and Retroviridae. Together, our data illustrate the utility of these 2 clinically approved compounds both as tools to identify host factors important in viral infection and as potential therapies against emerging viral infections.

Using advanced live cell imaging, we provide the first direct evidence, to our knowledge, that viral particles cotraffic intracellularly with AP complexes. Our imaging findings exclude a theory whereby AP complexes contribute to viral infections solely by recruiting or mediating intracellular traffic of host cargo components essential for the viral life cycle.

We show that sunitinib and erlotinib, potent, albeit nonselective, inhibitors of AAK1 and GAK, respectively, restrict DENV and EBOV infections in vitro and their combination reduces viremia, morbidity, and mortality in the relevant murine models. Replication assays demonstrating efficacy against viral species in 6 unrelated families (Table 1) further support our broad-spectrum hypothesis, though in vivo efficacy beyond dengue and Ebola remains to be tested. AAK1 and GAK have partially overlapping functions $(12,23)$, which may explain moderate antiviral effect in vitro with either sunitinib or erlotinib, yet synergistic activity upon treatment with both. The synergy also may result from inhibition of additional targets by these compounds. Although the observed reduction in DENV load was relatively modest in mice, it correlated with a significant survival benefit, comparable to the report on host $\alpha$-glucosidase inhibitor celgosivir (51), which yielded $100 \%$ protection from mortality with less than 10 -fold reduction in viremia. Importantly, the sunitinib/erlotinib combination remained protective in the mouse model of dengue even when administered after established infection, thereby supporting its promise as both prophylaxis and therapy.

We provide multiple lines of evidence to support modulation of AAK1 and GAK activity as an important mode of antiviral action of sunitinib and erlotinib in the dengue model. We demonstrate that these drugs inhibit both DENV entry and infectious virus production, analogous to the phenotype seen with RNAi-mediated suppression of clathrin-associated APs and AAK1 and GAK. Additionally, we demonstrate antiviral effects of more selective AAK1 and GAK inhibitors. Due to lack of affinity to most of sunitinib's and erlotinib's cancer targets (e.g., VEGFR and EGFR) $(28,35)$, the activity of the selective compounds further confirms that AAK1 and GAK are relevant antiviral targets. While the more selective GAK inhibitors also bind KIT, more work is required to validate whether KIT is an anti-DENV target. Furthermore, we characterized the mechanism by which the pharmacological inhibition of AAK1 and GAK mediates the anti-dengue effect. We establish that antiviral activity of sunitinib and erlotinib correlates with reduced phospho-AP2 levels in vitro. In accordance with this observation, we show that WT but not a phosphorylation AP2 mutant can rescue the anti-dengue effect of these drugs. We provide evidence that this mechanism also plays a role in vivo by demonstrating that antiviral activity correlates with reduced AP2 phosphorylation in tissues upon drug treatment. These findings reveal that a block in AP2 phosphorylation mechanistically explains at least in part the antiviral effect of AAK1 and GAK inhibitors. These findings also present AP2 phosphorylation as a useful pharmacodynamic biomarker in potential future clinical studies. We further demonstrate that sunitinib and erlotinib reduce HCV-AP1 and -AP2 cotrafficking by live cell imaging, thereby validating this mode of action at the molecular level. While AP-mediated intracellular membrane trafficking likely represents a primary mechanism by which AAK1 and GAK regulate viral infection, additional substrates of these kinases, such as NUMB, also may contribute to this function (23).

We explore the possible involvement of additional targets with $K_{\mathrm{D}}$ 's of $20 \mathrm{nM}$ or less reported for sunitinib and erlotinib using a siRNA library against 27 kinases. Our siRNA screen reveals that none of the other major targets of erlotinib beyond GAK, namely EGFR and STK10, affect DENV infection. In contrast, at least 3 additional targets of sunitinib beyond AAK1, namely AXL, KIT, and RET, may facilitate DENV infection and thus potentially also mediate sunitinib's antiviral effect. AXL is an already known attachment factor/signaling receptor for multiple RNA viruses, including DENV, EBOV, and possibly ZIKV (52-55). Although confirmatory studies with AXL-deficient cells are required, inhibition of AXL by sunitinib may contribute to its effect on DENV entry. KIT and RET are paralogs with no reported roles in RNA viral infections and await further investigation. Given our inability to silence expression of KIT without a substantial negative impact on cell viability (likely due to its role in cell survival and proliferation; ref. 56), its specific relevance to DENV infection remains unclear. Overall, our data underscore the utility of using sunitinib and erlotinib as pharmacological probes to identify novel host factors required for viral infection.

We speculate that inhibition of AAK1 and GAK accounts for these drugs' effect against a broad spectrum of viruses, particularly those previously shown to depend on AP1 and AP2 activity, such as EBOV (18, 37-39). Inhibition of additional kinases including AXL, KIT, and RET may play a role. Though most of the $\mathrm{EC}_{50}$ values we report fall in the low micromolar range for the 6 viral families tested (Table 1), the relative potency of sunitinib and erlotinib varies between the different viral species. Such differences can be attributed to both variations in the assays used and the likely distinct dependence on the various host factors targeted by these inhibitors in the life cycle of different viruses. Other mechanisms of action, such as modulation of immune responses, also could contribute to the protective phenotype observed in vivo.

Although toxicity is a concern when targeting host functions, finding a safe therapeutic window may be feasible. Sunitinib and erlotinib each are approved as a once-daily oral treatment for multiple cancers at doses comparable to those exhibiting antiviral activity in vivo. A combination therapy already has been evaluated clinically and was tolerated, albeit with an increase in the adverse events primarily related to gastrointestinal disturbances $(57,58)$. However, a shift from the long-term treatment of cancer to acute infection such as with DENV should improve tolerance and minimize adverse effects. The safety and efficacy of sunitinib and/or erlotinib will be evaluated in dengue patients in the near future and potentially in patients with EBOV disease in future outbreaks (ClinicalTrials.gov NCT02380625). 
The vast genetic diversity of viral species and replication strategies challenges the design of broadly effective direct-acting antivirals; however, a host-targeted approach could circumvent this issue. Sunitinib and erlotinib inhibit all 4 DENV serotypes. Moreover, a broad-spectrum therapy, such as with sunitinib/ erlotinib combination, could be used to treat DENV-CHIKV (59, 60) or DENV-ZIKV (61) coinfections and infections with newly emerging RNA viruses. It also can be administered even before an accurate diagnosis of a viral threat, thereby increasing protection. Furthermore, although viral resistant mutations can emerge during treatment with host-targeted approaches (62), targeting of host proteins that are not under the genetic control of viruses is more likely to have a higher barrier to resistance than classical direct-acting antivirals. This is exemplified by our data and treatment with cyclophilin inhibitors (63). We recognize, however, that our dengue resistance assay is somewhat limited by the shortterm virus passage. Although we predict that the genetic barrier to resistance is high, it may be possible to select for resistance over longer-term passage under different conditions or in a different, chronic infection model. Lastly, viruses use strategies similar to those of cancer cells for overcoming drug-mediated inhibition. Simultaneous inhibition of several kinases or targeting of several pathways by the same drug or drug combination may prove attractive in combating viral pathogens, as previously shown in cancer (64). Such "polypharmacology" by a single drug could increase the effectiveness while minimizing viral resistance.

In summary, our study serves as a proof of concept for the feasibility of identifying novel host-targeted broad-spectrum antiviral therapies via both repurposing and development of novel chemical entities. Such approaches may provide additive and possibly synergistic effects in combination with other strategies being developed to combat emerging viral infections.

\section{Methods}

Plasmids and virus constructs. ORFs encoding AP1M1 (AP1) and AP2M1 (AP2) were selected from the Human ORFeome library of cDNA clones (65) (Open Biosystems) and recombined into either pCherry (for mCherry fluorescence protein tagging) or pGLuc (for Gaussia Princeps luciferase fragment [GLuc] tagging) vectors using Gateway technology (Invitrogen). GFP-LC3 construct was previously described (66). pFLJ6/JFH(p7-Rluc2A) was a gift from Charles M. Rice (Rockefeller University, New York, New York, USA) (67). HCV TC-core was previously described (32). Plasmids used in the HCVpp entry assays (pNL4-3.Luc.R-E, pcDM8, and pcDM8-E1E2) were a gift from Shoshana Levy (Stanford University, Stanford, California, USA). DENV2 TSV01 Renilla reporter plasmid was a gift from Pei-Yong Shi (University of Texas Medical Branch, Galveston, Texas, USA) (68), and DENV 16681 plasmid (pD2IC-30P-NBX) used to produce virus for the plaque assay was a gift from Claire Huang (Centers for Disease Control and Prevention, Public Health Service, US Department of Health and Human Services, Fort Collins, Colorado, USA) (69). Additional DENV isolates and ZIKV MR766 were from ATCC/BEI Resources. Lentiviral constructs used for AP2 overexpression were cloned into the pRRLSIN backbone. rVSV-GP EBOV construct was a gift from Kartik Chandran (Albert Einstein College of Medicine, New York, New York, USA) (69, 70). Mouse-adapted N124D/K128E DENV2 PL046 was a gift from Sujan Shresta (70, 71). pCMV-DV2Rep was a gift from Andrew Yueh (Institute of Biotechnology and Pharmaceutical Research, Taipei, Taiwan) (34). Mutations were introduced by site-directed mutagenesis using the QuikChange kit (Stratagene).

Cells. Huh7 (Apath LLC), Huh7.5 (Apath LLC), BHK-21 (ATCC), and Vero (ATCC) cells were grown in DMEM (Mediatech) supplemented with $10 \%$ FBS, nonessential amino acids (Gibco), 1\% L-glutamine (Gibco), and 1\% penicillin-streptomycin (Gibco) and maintained in a humidified incubator with $5 \% \mathrm{CO}_{2}$ at $37^{\circ} \mathrm{C}$. C6/36 cells were grown in Leibovitz's L-15 media (CellGro) supplemented with 10\% FBS and 1\% HEPES in a humidified chamber at $28^{\circ} \mathrm{C}$ and $0 \% \mathrm{CO}_{2}$.

Reagents. The following reagents were used: sunitinib malate (Selleckchem), erlotinib (LC Laboratories), Captisol (Captisol), siImporter (Millipore), and Lipofectamine 2000 (Invitrogen). 12g and 12i were synthesized by the Herdewijn laboratory (28); 7737 and 7745 were synthesized by ACME Bioscience Inc.

Western blotting and antibodies. Cells were lysed in M-Per protein extraction reagent (Thermo Fisher Scientific). For phosphorprotein detection, cells were pretreated with $100 \mathrm{nM}$ calyculin A (Cell Signaling), a PP1 and PP2a phosphatase inhibitor, for 30 minutes prior to lysis. Liver tissue was homogenized in RIPA buffer supplemented with Halt protease and phosphatase inhibitor cocktail (Thermo Fisher Scientific) and $100 \mathrm{nM}$ calyculin A using 0.9- to 2-mm stainless steel beads in a BBX24 Bullet Blender homogenizer (NextAdvance). Clarified protein lysates were run on 4\%-12\% Bis-Tris gels (Invitrogen), transferred onto PVDF membranes (Bio-Rad). Blots were blocked and blotted with anti-AP1M1 (Abcam, catalog ab111135), anti-AP2M1 (Abcam, catalog ab75995), anti-GLuc (New England BioLabs, catalog E8023S), anti-phosphoAP2M1 (T156) (Cell Signaling, catalog 3843S), anti-AAK1 (Abcam, catalog ab134971), anti-GAK (MBL International, catalog MO573), and anti- $\beta$-actin (Sigma-Aldrich, catalog A3854) antibodies. Signal was detected with HRP-conjugated secondary antibodies. Band intensity was quantified with ImageJ software (NIH). See complete unedited blots in the supplemental material.

RNA interference. siRNAs (100-250 nM) were transfected into cells using silMPORTER (Millipore) 48 hours before infection. Sequences/catalog numbers are as follows: human AAK1 and GAK, Silencer Select predesigned siRNA ID\#s22494 and s5529, respectively (Thermo Fisher Scientific); Chlorocebus sabaeus (green monkey) AAK1 siRNA\#1, GGUAUAUGUUGGAACCAGATT; AAK1 siRNA\#2, GAAUAUUGUGGGUUACAUUTT; GAK SiRNA\#1, GCAUUAAAGAGGCUAUUAUTT; GAK siRNA\#2, CAGCAUCCAUAGGAAAAGATT; NT, Silencer Select negative control (Thermo Fisher Scientific, catalog 4390844). Infections were performed at 48 hours after transfection. AP1M1 and AP2M1 were silenced via transduction with shRNA-expressing lentivirus (TRCNO000218336, TRCN0000060239, or Mission pLKO.1-puro nonmammalian shRNA control; Sigma-Aldrich) and selection on $1 \mu \mathrm{g} / \mathrm{ml}$ puromycin. Custom Cherry-Pick ON-TARGETplus siRNA library against 27 kinase genes was purchased from Dharmacon (see Supplemental Table 1 for gene and siRNA sequence details).

Virus production. HCV 2a J6/JFH(p7-Rluc2A) was transcribed in vitro using Megascript T7 kit (Ambion), and DENV2 TSV01, 16681 (used for DENV in vitro assays), or N124D/K128E DENV2 PL046 (used for DENV in vivo infections) RNA was transcribed in vitro using mMessage/mMachine (Ambion) kits. HCVcc and HCVpp were produced as previously described (23). DENV was produced by electro- 
poration of RNA into BHK-21 cells, harvesting of supernatants at day 10 , and titering via standard plaque assays on BHK-21 cells. In parallel, on day 2 after electroporation, DENV-containing supernatant was used to inoculate C6/36 cells to amplify the virus. For in vivo experiments, DENV supernatant was concentrated 100 -fold by centrifugation at 50,000 g. rVSV-GP EBOV was propagated and titered on Vero cells via fluorescent-focus assay (69).

Entry assays. Huh7.5 cells were infected with $\operatorname{HCVpp}(71,72)$ and $8 \mu \mathrm{g} / \mathrm{ml}$ Polybrene for 4 hours. Firefly luciferase activity was measured at $48-72$ hours after infection. DENV2 entry was measured at 6 hours after infection of Huh7 cells by monitoring of Renilla luciferase activity. Luminescence was detected on InfiniteM1000 plate reader (Tecan). Vero cells were infected with rVSV-GP EBOV for 3 hours, total RNA was harvested and reverse transcribed, and EBOV GP transcript was quantified by real-time PCR and normalized to GAPDH expression.

Infection assays. Huh7 cells were infected with DENV or ZIKV in replicates $(n=3-10)$ for 4 hours at MOI of 0.01. Overall infection was measured either at 48 hours using a Renilla luciferase substrate or at 72 hours by plaque assays. Vero cells were infected with rVSV-GP EBOV for 4 hours and washed. At 20 hours after infection, cells were fixed with $4 \%$ formaldehyde and analyzed on an LSR II cytometer (BD Biosciences) using FITC channel. Data were processed using FlowJo software. EBOV infection was carried out under biosafety level 4 conditions. Forty-eight hours after infection, cells were formalin-fixed, and infection was measured by immunofluorescence using KZ52 anti-EBOV antibody in an Operetta HCS using the Harmony software package. Other viral infection assays, conducted by the Diamond lab (WNV), IBT Bioservices (CHIKV, RSV, JUNV), and the NIH/National Institute of Allergy and Infectious Diseases (others), were performed as summarized in Table 1.

$R N A$ replication assays. HCV RNA replication was measured 72 hours after electroporation, as previously described (22). DENV2 replication assays were performed as previously described (34). Briefly, Huh7 cells were transfected with DNA-launched DENV2 replicon, pCMV-DV2Rep along with TET-ON plasmids. Thirty-six hours after transfection, viral RNA transcription was induced by doxycycline and shut down by changing to doxycycline-free medium 6 hours later. Replication was monitored by luciferase activity every day for 3 days. Data were normalized to activity at 24 hours after induction.

Infectious virus production. Infectious HCV or DENV production (i.e., extracellular infectivity) was measured in culture supernatants of cells electroporated with viral RNA for 48-72 hours and used to infect naive cells for 48 hours. Intracellular HCV infectivity was measured by inoculation of naive cells with lysates of electroporated cells subjected to 4 rounds of free-thawing and clarified at 5,000 $\mathrm{g}$, as previously described (22).

Pharmacological inhibition. For entry assays, cells were pretreated with the inhibitors or DMSO for 1 hour before and for the duration of the infection, followed by replacement with drug-free medium. For the overall infection, RNA replication, or infectious virus production assays, inhibitors were left in for the duration of the study.

Gain-of-function assays. WT or T156A AP2 or empty vector control was expressed ectopically in Huh7 cells by lentiviral transduction. Twenty-four hours after transduction, cells were pretreated with sunitinib and erlotinib, infected with luciferase reporter DENV at MOI of 0.01 , and incubated for 72 hours prior to luciferase and viability assays.
Live cell imaging. Huh7.5 cells were infected with concentrated HCV TC-core (32) at MOI of 1 for 24 hours, then transfected with either AP1- or AP2-mCherry using Lipofectamine 2000 (Invitrogen) and seeded onto collagen-coated 35-mm fluorodishes (World Precision Instruments). At 72 hours after infection, cells were labeled with biarsenical dye $(1.25 \mu \mathrm{M})$ in Opti-MEM at $37^{\circ} \mathrm{C}$ for 30 minutes, then washed 3 times with $1 \mathrm{X}$ BAL (2,3-dimercapto-1-propanol) wash buffer (Invitrogen) supplemented with $500 \mu \mathrm{M}$ 1,2-ethanedithiol in OptiMEM. The cells were washed and incubated in prewarmed imaging media (DMEM-F12; Invitrogen) supplemented with 10\% FBS, $0.1 \mathrm{mM}$ nonessential amino acids, $1 \%$ penicillin-streptomycin, and $25 \mathrm{mM}$ HEPES. When specified, TC-core-infected cells were incubated with DMSO, sunitinib $(4 \mu \mathrm{M})$, or erlotinib $(10 \mu \mathrm{M})$ beginning at 24 hours after infection. Time-lapse images were taken using a Leica SP5 II AOBS Tandem Scanner Spectral confocal microscope with a $\times 100$ 1.46 oil objective and a heated $\left(37^{\circ} \mathrm{C}\right)$ chamber. An average of 5 movies representing individual cells with 15-30 trackable puncta each were recorded per sample with sequential frames taken every $2 \mathrm{sec}$ onds. Individual core puncta run lengths and transport velocities were calculated using the Manual Tracking plug-in for ImageJ, measuring the distance traveled (in any direction) between frames for a respective TC-core puncta.

Resistance studies. DENV4 (BC287/97; ATCC/BEI Resources, BEI NR-3806) was used to inoculate Huh7 cells at MOI of 0.01 and passaged every 3 days by transferring of an equal volume of viral supernatant to naive cells under increasing drug selection $(0.5-1.5 \mu \mathrm{M}$, passages 1-6; $2.5 \mu \mathrm{M}$, passages 7 and 8). Upon completion of 8 passages, virus from the resulting supernatants was titered by plaque assays. SDM25N resistance mutation in NS4B at passage 8 was confirmed by purification and reverse transcription of viral RNA from supernatants of cells treated with DMSO or SDM25N as described in the RNA extraction and quantification section. NS4B region was amplified with iProof high-fidelity PCR kit (Bio-Rad) using primers NS4B forward GATGTGGAYYTGAGACCAGCYTCAGCATGGAC and NS4B reverse AGTCAANACTTCACAGAAAGCCCATGTTGTTCTCATCAA $(\mathrm{N}=$ any base, $\mathrm{Y}=\mathrm{C}$ or $\mathrm{T}$ ) and sequenced (Sequetech Corp.).

In vivo dengue studies. AG-129 mice deficient in type I and II IFN receptors were obtained from Harry Greenberg (Stanford University, Stanford, California, USA). Mice were backcrossed to C57B6/6J for $n=10$ generations to obtain congenic AG-B6 strain. Age-matched, male and female mice at $8-10$ weeks of age were used for all experiments. Mouse-adapted N124D/K128E DENV2 $\left(5 \times 10^{5} \mathrm{PFU}\right.$ produced in BHK-21 cells or $10^{7} \mathrm{PFU}$ produced in $\mathrm{C} 6 / 36$ cells) was inoculated retro-orbitally into mice under general and local anesthesia. Drugs were administered at the indicated doses in a total volume of 100-200 $\mu \mathrm{l}$ per animal using $10 \%$ Captisol as vehicle. Drugs or vehicle were administered i.p. or orally once or twice daily starting at the time of inoculation or at various time points after inoculation for a total of 3-5 days. Mice were monitored twice daily until the conclusion of the experiment. Moribund animals were euthanized by carbon dioxide inhalation. Serum was isolated from whole blood harvested retroorbitally at 48 or 72 hours after DENV inoculation under general and local anesthesia. Tissues were harvested following euthanasia and snap-frozen on dry ice until further analysis.

In vivo Ebola studies. Groups of 10 female C57BL/6 mice (8-12 weeks of age) purchased from Jackson Laboratory were treated with drugs or vehicle 6 hours before viral challenge. Erlotinib and sunitinib 
were administered i.p. at the indicated doses in a total volume of 200 $\mu \mathrm{l}$ using 10\% Captisol as vehicle. A group of 10 mice was left untreated to allow for interpretation of any survival seen in the vehicle-treated group. Mice were inoculated i.p. with 100 PFU of mouse-adapted EBOV. Mice were then treated once daily for a total of 10 days and observed daily for 28 days for lethality or clinical signs of disease.

RNA extraction and quantification. Total RNA from cells was isolated using an RNA purification kit (Macherey-Nagel). Mouse serum was purified from whole blood using Terumo Capiject Capillary Blood collection tubes (Thermo Fisher Scientific). Tissues were homogenized using 0.9- to 2-mm stainless steel beads in a BBX24 Bullet Blender homogenizer (NextAdvance). Total RNA from serum and tissues was extracted with QIAamp UltraSens Virus kit (Qiagen). cDNA was generated using a high-capacity cDNA reverse transcription kit (Invitrogen). DENV RNA was quantified by qRT-PCR using TaqMan GEX master mix (Thermo Fisher Scientific), and AAK1, GAK, EBOV GP, and housekeeping gene (GAPDH) RNA was quantified using iTaq Universal SYBR Green Supermix (Bio-Rad) on a StepOnePlus real-time PCR system (Applied Biosystems). Primer and probe sequences are as follows: DENV2 forward, GAGAGCAGATCTCTGATGAATAA; DENV2 reverse, ACTGTTGCACAGTCGACAC; DENV2 probe, TATGCTGAAACGCGAGAGAAACCGC; GAP$D H$ forward, GAAATCCCATCACCATCTTCCAG; GAPDH reverse, GAGCCCCAGCCTTCTCCATG.

Viability assays. Viability was assessed using alamarBlue reagent (Invitrogen) according to the manufacturer's protocol. Fluorescence was detected at $560 \mathrm{~nm}$ on an InfiniteM1000 plate reader (Tecan).

Data analysis of combination drug treatment. Synergy/antagonism analysis was performed using the MacSynergy II program as previously described $(23,73)$. Matrix data sets in 4 replicates were assessed at the 95\% confidence level for each experiment. Synergy and log volume were calculated. As suggested by Prichard et al. (73), such data sets should be interpreted as follows: volumes of synergy or antagonism at values less than $25 \mu \mathrm{M}^{2} \%$ are insignificant, those of $25-50 \mu \mathrm{M}^{2} \%$ are minor but significant, those of $50-100 \mu \mathrm{M}^{2} \%$ are moderate and probably important in vivo, and those of greater than $100 \mu \mathrm{M}^{2} \%$ are strong and likely to be important in vivo.

Statistics. All data were analyzed with GraphPad Prism software. Fifty percent effective concentrations $\left(\mathrm{EC}_{50}\right)$ were measured by fitting of data to a 3-parameter logistic curve. $P$ values were calculated by 2 -tailed unpaired $t$ test and 1- or 2-way ANOVA with either Dunnett's or Tukey's multiple comparisons tests for in vitro data sets and by Mann-Whitney test for in vivo data sets as specified in each figure legend. Survival curve $P$ values were calculated by log-ranked Mantel-Cox test.

Study approval. Animal research was conducted under a protocol approved by Stanford's IACUC (Administrative Panel on Laboratory Animal Care) and its Institutional Biosafety Committee (Administrative Panel on Biosafety) or by the US Army Medical Research Institute of Infectious Diseases (USAMRIID) IACUC in compliance with the Animal Welfare Act and other federal statutes and regulations relating to animals and experiments involving animals. The Stanford and USAMRIID animal facilities are AAALAC-accredited and adhere to the principles stated in the 2005 Guide for the Care and Use of Laboratory Animals (National Academies Press). DENV and EBOV challenge studies were conducted in a biosafety level 2 (BSL-2) and a maximum-containment BSL-4 facility, respectively. Moribund mice were humanely euthanized on the basis of IACUC-approved criteria.

\section{Author contributions}

SE, EB, GN, AS, SYP, JB, RBB, JG, MSD, JMD, and GR conceived and designed the experiments. EB, GN, AS, SYP, JB, SW, FX, RBB, JG, RRB, and CMN performed the experiments. SE, EB, GN, AS, SYP, JB, SW, FX, RBB, JG, MSD, JMD, and GR analyzed the data. $\mathrm{RM}, \mathrm{CMN}, \mathrm{SD}$, and $\mathrm{PH}$ contributed reagents/materials/models. $\mathrm{EB}$ and SE wrote the manuscript. SE supervised the overall project.

\section{Acknowledgments}

This work was supported by award 1U19 AI10966201 (Centers of Excellence for Translational Research) from the National Institute of Allergy and Infectious Diseases (NIAID) to SE, NIAID U19 AI083019 to MSD, grant RSG-14-11 0-0 1-MPC from the American Cancer Society to SE and GR, and grants from Stanford Bio-X, the Stanford SPARK program, and the Stanford Translational Research and Applied Medicine program. In vitro studies were also supported by grant 2013100 from the Doris Duke Charitable Foundation and grant PR151090 from the Department of Defense office of the Congressionally Directed Medical Research Programs. GN was supported by the Child Health Research Institute, Lucile Packard Foundation for Children's Health, as well as the Stanford Clinical and Translational Science Award (CTSA, grant UL1 TR000093). SYP was supported by Taiwan Ministry of Science and Technology grant 103-2917-I-564-033. JMD's work acknowledges the support of the Joint Science and Technology Office-Defense Threat Reduction Agency (CB3958) in completion of the live virus experiments. The opinions, interpretations, conclusions, and recommendations are those of the authors and are not necessarily endorsed by the US Army or the other funders. We thank Scott Weaver and Robert Tesh for the ZIKV (MR766), Mark N. Prichard for providing the MacSynergy II program, Kartik Chandran for the rVSV-GP EBOV construct, Eva Harris and Karla Kirkegaard for assistance with establishing dengue tissue culture and mouse models in our laboratory, and Alex McMillan (with support from CTSA UL1 TR001085) for assistance with the statistical analysis.

Address correspondence to: Shirit Einav, Department of Medicine, Stanford University, 300 Pasteur Drive, Lane Building L127, Stanford, California 94305, USA. Phone: 650.723.8656; E-mail: seinav@stanford.edu.

GN's present address is: International Center for Infectiology Research, Université de Lyon; Inserm, U1111; Ecole Normale Supérieure de Lyon; Université Claude Bernard Lyon 1, Centre International de Recherche en Infectiologie; LabEx Ecofect, Université de Lyon, Lyon, France.
1. Bhatt $S$, et al. The global distribution and burden of dengue. Nature. 2013;496(7446):504-507.

2. Flipse J, Smit JM. The complexity of a

Dengue vaccine: a review of the human antibody response. PLoS Negl Trop Dis. 2015;9(6):e0003749.

3. Priyamvada L, et al. Human antibody responses after dengue virus infection are highly cross- reactive to Zika virus. Proc Natl Acad Sci U S A. 2016;113(28):7852-7857.

4. Dejnirattisai W, et al. Dengue virus sero-crossreactivity drives antibody-dependent enhance- 
ment of infection with Zika virus. Nat Immunol. 2016;17(9):1102-1108.

5. Stettler K, et al. Specificity, cross-reactivity, and function of antibodies elicited by Zika virus infection. Science. 2016;353(6301):823-826.

6. Henao-Restrepo AM, et al. Efficacy and effectiveness of an rVSV-vectored vaccine expressing Ebola surface glycoprotein: interim results from the Guinea ring vaccination cluster-randomised trial. Lancet. 2015;386(9996):857-866.

7. Bekerman E, Einav S. Infectious disease. Combating emerging viral threats. Science. 2015;348(6232):282-283.

8. Park SY, Guo X. Adaptor protein complexes and intracellular transport. Biosci Rep. 2014;34(4):e00123.

9. Olusanya O, Andrews PD, Swedlow JR, Smythe E. Phosphorylation of threonine 156 of the mu2 subunit of the AP2 complex is essential for endocytosis in vitro and in vivo. Curr Biol. 2001;11(11):896-900.

10. Ricotta D, Conner SD, Schmid SL, von Figura K, Honing S. Phosphorylation of the AP2 mu subunit by AAK1 mediates high affinity binding to membrane protein sorting signals. J Cell Biol. 2002;156(5):791-795.

11. Umeda A, Meyerholz A, Ungewickell E. Identification of the universal cofactor (auxilin 2) in clathrin coat dissociation. Eur J Cell Biol. 2000;79(5):336-342.

12. Zhang CX, Engqvist-Goldstein AE, Carreno S, Owen DJ, Smythe E, Drubin DG. Multiple roles for cyclin G-associated kinase in clathrin-mediated sorting events. Traffic. 2005;6(12):1103-1113.

13. Ghosh P, Kornfeld S. AP-1 binding to sorting signals and release from clathrin-coated vesicles is regulated by phosphorylation. J Cell Biol. 2003;160(5):699-708.

14. Conner SD, Schmid SL. Identification of an adaptor-associated kinase, AAK1, as a regulator of clathrin-mediated endocytosis. J Cell Biol. 2002;156(5):921-929.

15. Fingerhut A, von Figura K, Honing S. Binding of AP2 to sorting signals is modulated by AP2 phosphorylation. J Biol Chem. 2001;276(8):5476-5482.

16. Lee DW, Zhao X, Zhang F, Eisenberg E, Greene LE. Depletion of GAK/auxilin 2 inhibits receptormediated endocytosis and recruitment of both clathrin and clathrin adaptors. J Cell Sci. 2005;118(pt 18):4311-4321.

17. Alconada A, Bauer U, Hoflack B. A tyrosine-based motif and a casein kinase II phosphorylation site regulate the intracellular trafficking of the varicella-zoster virus glycoprotein I, a protein localized in the trans-Golgi network. EMBO J. 1996;15(22):6096-6110.

18. Bhattacharyya S, Hope TJ, Young JA. Differential requirements for clathrin endocytic pathway components in cellular entry by Ebola and Marburg glycoprotein pseudovirions. Virology. 2011;419(1):1-9.

19. Dutta D, et al. EphrinA2 regulates clathrin mediated KSHV endocytosis in fibroblast cells by coordinating integrin-associated signaling and c-Cbl directed polyubiquitination. PLoS Pathog. 2013;9(7):e1003510.

20. Huang HC, Chen CC, Chang WC, Tao MH,
Huang C. Entry of hepatitis B virus into immortalized human primary hepatocytes by clathrin-dependent endocytosis. J Virol. 2012;86(17):9443-9453.

21. Humphries AC, Dodding MP, Barry DJ, Collinson LM, Durkin CH, Way M. Clathrin potentiates vaccinia-induced actin polymerization to facilitate viral spread. Cell Host Microbe. 2012;12(3):346-359.

22. Neveu G, Barouch-Bentov R, Ziv-Av A, Gerber D, Jacob Y, Einav S. Identification and targeting of an interaction between a tyrosine motif within hepatitis $\mathrm{C}$ virus core protein and AP2M1 essential for viral assembly. PLoS Pathog. 2012;8(8):e1002845.

23. Neveu G, Ziv-Av A, Barouch-Bentov R, Berkerman E, Mulholland J, Einav S. AP-2-associated protein kinase 1 and cyclin $\mathrm{G}$-associated kinase regulate hepatitis $C$ virus entry and are potential drug targets. JVirol. 2015;89(8):4387-4404.

24. Ohka S, Ohno H, Tohyama K, Nomoto A. Basolateral sorting of human poliovirus receptor alpha involves an interaction with the mu1B subunit of the clathrin adaptor complex in polarized epithelial cells. Biochem Biophys Res Commun. 2001;287(4):941-948.

25. Ohno H, Aguilar RC, Fournier MC, Hennecke S, Cosson P, Bonifacino JS. Interaction of endocytic signals from the HIV-1 envelope glycoprotein complex with members of the adaptor medium chain family. Virology. 1997;238(2):305-315.

26. Agrawal T, Schu P, Medigeshi GR. Adaptor protein complexes- 1 and 3 are involved at distinct stages of flavivirus life-cycle. Sci Rep. 2013;3:1813.

27. Karaman MW, et al. A quantitative analysis of kinase inhibitor selectivity. Nat Biotechnol. 2008;26(1):127-132.

28. Kovackova S, et al. Selective inhibitors of cyclin G Associated Kinase (GAK) as anti-hepatitis C agents. J Med Chem. 2015;58(8):3393-3410.

29. Benedicto I, et al. Clathrin mediates infectious hepatitis $\mathrm{C}$ virus particle egress. J Virol. 2015;89(8):4180-4190.

30. Coller KE, Heaton NS, Berger KL, Cooper JD, Saunders JL, Randall G. Molecular determinants and dynamics of hepatitis $\mathrm{C}$ virus secretion. PLoS Pathog. 2012;8(1):e1002466.

31. Sorensen EB, Conner SD. AAK1 regulates Numb function at an early step in clathrin-mediated endocytosis. Traffic. 2008;9(10):1791-1800.

32. Coller KE, Heaton NS, Berger KL, Cooper JD, Saunders JL, Randall G. Molecular determinants and dynamics of hepatitis $\mathrm{C}$ virus secretion. PLoS Pathog. 2012;8(1):e1002466.

33. Wacker I, Kaether C, Krömer A, Migala A, Almers W, Gerdes HH. Microtubule-dependent transport of secretory vesicles visualized in real time with a GFP-tagged secretory protein. J Cell Sci. 1997;110(pt 13):1453-1463.

34. Yang CC, et al. Characterization of an efficient dengue virus replicon for development of assays of discovery of small molecules against dengue virus. Antiviral Res. 2013;98(2):228-241.

35. Kostich $\mathrm{W}$ et al. Inhibition of AAK1 kinase as a novel therapeutic approach to treat neuropathic pain. JPharmacol Exp Ther. 2016;358(3):371-386.

36. van Cleef $\mathrm{KW}$, et al. Identification of a new den- gue virus inhibitor that targets the viral NS4B protein and restricts genomic RNA replication. Antiviral Res. 2013;99(2):165-171.

37. Bhattacharyya S, Mulherkar N, Chandran K. Endocytic pathways involved in filovirus entry: advances, implications and future directions. Viruses. 2012;4(12):3647-3664.

38. Poirier S, et al. The cytosolic adaptor AP-1A is essential for the trafficking and function of Niemann-Pick type C proteins. Traffic. 2013;14(4):458-469.

39. Carette JE, et al. Ebola virus entry requires the cholesterol transporter Niemann-Pick C1. Nature. 2011;477(7364):340-343.

40. Johnson AJ, Roehrig JT. New mouse model for dengue virus vaccine testing. JVirol. 1999;73(1):783-786.

41. Schul W, Liu W, Xu HY, Flamand M, Vasudevan SG. A dengue fever viremia model in mice shows reduction in viral replication and suppression of the inflammatory response after treatment with antiviral drugs. J Infect Dis. 2007;195(5):665-674.

42. Nair AB, Jacob S. A simple practice guide for dose conversion between animals and human. JBasic Clin Pharm. 2016;7(2):27-31.

43. Mendel DB, et al. In vivo antitumor activity of SU11248, a novel tyrosine kinase inhibitor targeting vascular endothelial growth factor and platelet-derived growth factor receptors: determination of a pharmacokinetic/pharmacodynamic relationship. Clin Cancer Res. 2003;9(1):327-337.

44. Higgins B, et al. Antitumor activity of erlotinib (OSI-774, Tarceva) alone or in combination in human non-small cell lung cancer tumor xenograft models. Anticancer Drugs. 2004;15(5):503-512.

45. Haznedar JO, et al. Single- and multiple-dose disposition kinetics of sunitinib malate, a multitargeted receptor tyrosine kinase inhibitor: comparative plasma kinetics in non-clinical species. Cancer Chemother Pharmacol. 2009;64(4):691-706.

46. Chee EL, Lim AY, Modamio P, FernandezLastra C, Segarra I. Sunitinib tissue distribution changes after coadministration with ketoconazole in mice. Eur J Drug Metab Pharmacokinet. 2016;41(3):309-319.

47. Zerbe LK, et al. Inhibition by erlotinib of primary lung adenocarcinoma at an early stage in male mice. Cancer Chemother Pharmacol. 2008;62(4):605-620.

48. Orozco S, et al. Characterization of a model of lethal dengue virus 2 infection in C57BL/6 mice deficient in the alpha/beta interferon receptor. $J$ Gen Virol. 2012;93(pt 10):2152-2157.

49. Human Kinome Heat Map. SuperNova Life Science. http://www.supernovalifescience.com/ HM/HM\%2041.pdf. Accessed January 11, 2017.

50. Davis MI, et al. Comprehensive analysis of kinase inhibitor selectivity. Nat Biotechnol. 2011;29(11):1046-1051.

51. Rathore AP, et al. Celgosivir treatment misfolds dengue virus NS1 protein, induces cellular pro-survival genes and protects against lethal challenge mouse model. Antiviral Res. 2011;92(3):453-460.

52. Brindley MA, et al. Tyrosine kinase receptor Axl enhances entry of Zaire ebolavirus without direct interactions with the viral glycoprotein. Virology. 
2011;415(2):83-94.

53. Meertens L, et al. The TIM and TAM families of phosphatidylserine receptors mediate dengue virus entry. Cell Host Microbe. 2012;12(4):544-557.

54 . Hamel R, et al. Biology of Zika virus infection in human skin cells. JVirol. 2015;89(17):8880-8896.

55. Nowakowski TJ, Pollen AA, Di Lullo E, SandovalEspinosa C, Bershteyn M, Kriegstein AR. Expression analysis highlights AXL as a candidate Zika virus entry receptor in neural stem cells. Cell Stem Cell. 2016;18(5):591-596.

56. Roskoski R. Signaling by Kit protein-tyrosine kinase--the stem cell factor receptor. Biochem Biophys Res Commun. 2005;337(1):1-13.

57. Scagliotti GV, et al. Sunitinib plus erlotinib versus placebo plus erlotinib in patients with previously treated advanced non-small-cell lung cancer: a phase III trial. JClin Oncol. 2012;30(17):2070-2078.

58. Blumenschein GR, et al. Sunitinib plus erlotinib for the treatment of advanced/metastatic nonsmall-cell lung cancer: a lead-in study. J Thorac Oncol. 2012;7(9):1406-1416.

59. Chipwaza B, et al. Dengue and Chikungunya fever among viral diseases in outpatient febrile children in Kilosa district hospital, Tanzania. PLoS Negl Trop Dis. 2014;8(11):e3335.
60. Karthik R, Vineetha KR, Raut CG. Seroprevalance of dengue and chikungunya co infection and its clinical correlation in bangalore city hospitals. Int J Curr Res. 2014;6(12):11040-11044.

61. Dupont-Rouzeyrol M, et al. Co-infection with Zika and dengue viruses in 2 patients, New Caledonia, 2014. Emerging Infect Dis. 2015;21(2):381-382.

62. de Wispelaere M, LaCroix AJ, Yang PL. The small molecules AZD0530 and dasatinib inhibit dengue virus RNA replication via Fyn kinase. J Virol. 2013;87(13):7367-7381.

63. Lin K, Gallay P. Curing a viral infection by targeting the host: the example of cyclophilin inhibitors. Antiviral Res. 2013;99(1):68-77.

64. Knight ZA, Lin H, Shokat KM. Targeting the cancer kinome through polypharmacology. Nat Rev Cancer. 2010;10(2):130-137.

65. Rual JF, et al. Human ORFeome version 1.1: a platform for reverse proteomics. Genome Res. 2004;14(10B):2128-2135.

66. Heaton NS, Randall G. Dengue virus-induced autophagy regulates lipid metabolism. Cell Host Microbe. 2010;8(5):422-432.

67. Tscherne DM, Jones CT, Evans MJ, Lindenbach BD, McKeating JA, Rice CM. Time- and temperature-dependent activation of hepatitis
C virus for low-pH-triggered entry. J Virol. 2006;80(4):1734-1741.

68. Zou G, Xu HY, Qing M, Wang QY, Shi PY. Development and characterization of a stable luciferase dengue virus for high-throughput screening. Antiviral Res. 2011;91(1):11-19.

69. Huang CY, et al. The dengue virus type 2 envelope protein fusion peptide is essential for membrane fusion. Virology. 2010;396(2):305-315.

70. Chandran K, Sullivan NJ, Felbor U, Whelan SP, Cunningham JM. Endosomal proteolysis of the Ebola virus glycoprotein is necessary for infection. Science. 2005;308(5728):1643-1645.

71. Prestwood TR, Prigozhin DM, Sharar KL, Zellweger RM, Shresta S. A mouse-passaged dengue virus strain with reduced affinity for heparan sulfate causes severe disease in mice by establishing increased systemic viral loads. J Virol. 2008;82(17):8411-8421.

72. Bartosch B, Dubuisson J, Cosset FL. Infectious hepatitis $\mathrm{C}$ virus pseudo-particles containing functional E1-E2 envelope protein complexes. JExp Med. 2003;197(5):633-642.

73. Prichard MN, Shipman C. Analysis of combinations of antiviral drugs and design of effective multidrug therapies. Antivir Ther (Lond). 1996;1(1):9-20. 